\title{
Integration eines digitalen Assistenzsystems für die industrielle Montage
}

\author{
Thimo Keller, Christian Bayer, Joachim Metternich, Mehrach Saki, \\ Stephanie Schmidt, Oliver Sträter, Wolfgang Anlauft \\ und Hans-Dieter Hartwich
}

\section{Zusammenfassung}

Dieser Beitrag beschreibt das Forschungsprojekt IntAKom (Intelligente Aufwertung der manuellen und teilautomatisierten Arbeit durch den Einsatz digitaler Kommunikationstechnologie) welches in Zusammenarbeit zwischen Forschung und Industrie konstruktive Ansätze guter digitaler Assistenz in der Produktion entwickelt und erprobt. Die Vorgehensweise wird an einem Anwendungsbeispiel aufgezeigt. Es wird beschrieben, wie das digitale Assistenzsystem in das Arbeitsumfeld integriert wurde. Gerade bei einer hohen Produktvielfalt erhöht sich die Komplexität

T. Keller $\cdot$ C. Bayer $(\bowtie) \cdot$ J. Metternich

Institut für Produktionsmanagement, Technologie und Werkzeugmaschinen, Technische Universität Darmstadt, Darmstadt, Deutschland

E-Mail: c.bayer@PTW.TU-Darmstadt.de; t.keller@PTW.TU-Darmstadt.de; j.metternich@PTW.TU-Darmstadt.de

M. Saki · S. Schmidt · O. Sträter

Fachgebiet Arbeits- und Organisationspsychologie, Universität Kassel,

Kassel, Deutschland

E-Mail: m.saki@uni-kassel.de; stephanie.schmidt@uni-kassel.de;

oliver.straeter@uni-kassel.de

W. Anlauft · H.-D. Hartwich

ffw GmbH - Gesellschaft für Personal- und Organisationsentwicklung,

Nürnberg, Deutschland

E-Mail: anlauft@ ffw-nuernberg.de; hartwich@ @ffw-nuernberg.de

(C) Der/die Autor(en) 2021

T. Jeske und F. Lennings (Hrsg.), Produktivitätsmanagement 4.0, ifaa-Edition, https://doi.org/10.1007/978-3-662-61584-3_5 
in der Produktion. Die Beschäftigten können so durch den Einsatz digitaler Assistenzsysteme bei der Bewältigung der steigenden Anforderungen unterstützt werden. Darüber hinaus wird die Vorgehensweise zur Zielerreichung aus Sicht der Prozess-, Beschäftigten-, und Organisationsebene dargestellt und erste Projektergebnisse aufgezeigt. Auch die Erfolgsfaktoren für die Einführung eines digitalen Assistenzsystems werden abschließend in Form von Lessons learned formuliert.

\subsection{Anwendungsbeispiel}

Aufgrund sinkender Losgrößen und einer steigenden Produktvarianz stehen viele Unternehmen vor der Herausforderung, flexibel und schnell auf Kundenwünsche zu reagieren. Um diese Flexibilität zu erreichen, müssen Arbeitsbedingungen, technische Prozesse und die Arbeitsorganisation verbessert bzw. angepasst werden. Die Umsetzung kann durch digitale Lösungen erfolgen. Abb. 5.1 stellt die Herausforderungen und die Erreichung der Ziele durch digitale Assistenz exemplarisch für ein Verbundunternehmen des IntAKom-Projekts dar.

Das Verbundunternehmen verfügt über ein umfangreiches Produktportfolio, um individuelle und energieeffiziente Lösungen auf dem Markt anzubieten. Aufgrund der Vielfalt von Produktbaureihen wurde ein digitales Assistenzsystem für den Montagearbeitsplatz eingeführt, um die Qualität der Produkte sicherzustellen und schnell auf Kundenwünsche reagieren zu können. Außerdem wird angestrebt, die Beschäftigten bei der resultierenden Komplexität aufgrund der Variantenvielfalt im Bereich der Montage zu unterstützen. Ein weiteres Ziel der Digitalisierungsmaßnahmen im Bereich der Endmontage ist die effizientere Gestaltung der technischen Prozesse, um dadurch z. B. unproduktive Aufgaben zu reduzieren und die Kommunikation mit anderen Abteilungen durch besser vernetzte Schnittstellen zu erhöhen.

Das digitale Assistenzsystem unterstützt die Beschäftigten bei der Vormontage und der Prüfung bis hin zum Verpacken des Produktes. Die Montageinsel ist in U-Form aufgebaut und mit verschiedenen Technologien ausgestattet. Berührungsbildschirme, Tablets, kabellose Handscanner und eine moderne Prüftechnik unterstützen die Beschäftigten in ihrer Tätigkeit. Das digitale Assistenzsystem ist direkt mit dem ERP-System verbunden und bezieht darüber die Daten zu den Aufträgen. Hier können die Beschäftigten ihre Aufträge eigenständig direkt am Arbeitsplatz auswählen. Bereits bei der Vorbereitung/beim Rüsten des Arbeitsplatzes unterstützt das Assistenzsystem. Eingesetzt werden Handscanner, um eine digitale Stückliste abzuarbeiten. Die digitale Stückliste wird beim Buchen des Auftrags direkt im System zur Verfügung gestellt. Die Beschäftigten können die benötigten Teile des Auftrags direkt vor Ort abscannen und so unnötige Suchprozesse durch 


\begin{tabular}{|c|c|c|}
\hline Ausgangssituation & & Zielzustand \\
\hline $\begin{array}{l}\text { - Kleinere Losgrößen führen zu } \\
\text { einer wachsenden } \\
\text { Produktvarianz } \\
\text { - Flexible und schnelle Reaktion } \\
\text { auf Kundenwünsche erforderlich } \\
\text { - Hoher Aufwand bei der } \\
\text { Einarbeitung saisonal } \\
\text { Beschäftigter }\end{array}$ & $\begin{array}{l}\text { Digitale Assistenz } \\
\text { - Direkte Kopplung an das ERP- } \\
\text { System } \\
\text { - Handscanner zum mobilen } \\
\text { Kommissionieren anhand der } \\
\text { Stückliste } \\
\text { - Unterstützung durch } \\
\text { Informationen auf Anforderung } \\
\text { (Montagepläne/-videos usw.) } \\
\text { - Führung durch das digitale } \\
\text { Assistenzsystem bei qualitäts- } \\
\text { /sicherheitsrelevanten } \\
\text { Tätigkeiten } \\
\text { - Visuelle Rückmeldung der } \\
\text { Ergebnisse bei Verschraubungen } \\
\text { und Prüfungen } \\
\text { - Störungen können direkt } \\
\text { eingetragen werden } \\
\text { - Suchfunktion für bereits } \\
\text { eingetragene Störungen mit } \\
\text { Lösungsbeispielen }\end{array}$ & $\begin{array}{l}\text { - Erhöhte Komplexität } \\
\text { beherrschbar machen } \\
\text { - Prozesse effizienter gestalten } \\
\text { - Effizientere Vernetzung der } \\
\text { Abteilungen } \\
\text { - Schnelleres Rüsten } \\
\text { - Fehlerreduzierung } \\
\text { - Schnellerer Zugriff auf } \\
\text { Informationen } \\
\text { - Qualitätssicherung in der } \\
\text { Montage } \\
\text { - Schnellere Einarbeitung neuer } \\
\text { Mitarbeiter } \\
\text { - Verbesserte Zusammenarbeit } \\
\text { unter den Mitarbeitern }\end{array}$ \\
\hline
\end{tabular}

Abb. 5.1 Herausforderungen und Erreichung der Ziele durch den Einsatz eines digitalen Assistenzsystems. (Eigene Darstellung)

fehlerhaftes und/oder Übersehen von Teilen vermeiden, da das System die gescannten und die noch ausstehenden Teile anzeigt. Während der Montage kann das System die Beschäftigten unterstützen, führt diese jedoch im Sinne eines klassischen Werkerführungssystems ausschließlich bei qualitätskritischen Prozessschritten. Die Beschäftigten können sich die Montageschritte vor Ort anzeigen lassen und werden vom Assistenzsystem nicht durch jeden einzelnen Schritt geführt. Dadurch soll vermieden werden, dass die Tätigkeiten Schritt für Schritt vorgegeben und somit kleinteiliger und monotoner werden. Alle benötigten Informationen, wie Montagepläne, Montagevideos usw., sind im System hinterlegt und können direkt vor Ort abgerufen werden. Hierdurch wird eine schnellere Einarbeitung der Beschäftigten bei neuen Produkten ermöglicht. Aber auch bei neuen Beschäftigten können so schnellere Einarbeitungszeiten erzielt werden.

Die Schraubprogramme werden zu Beginn des Auftrags eingestellt und die Ergebnisse der qualitätskritischen Verschraubungen in Echtzeit angezeigt und in einer Datenbank hinterlegt. Auch bei der Prüfung werden die Beschäftigten durch Informationen über den Prüfprozess und den Prüfstatus direkt am Arbeitsplatz durch eine visuelle Anzeige geführt, da diese Schritte sicherheits- und qualitätsrelevant sind. Durch die visuelle Rückmeldung und die Sicherung der Schrauben- und Prüfergebnisse können keine qualitäts- und sicherheitsrelevanten Fehler auf die Beschäftigten zurückgeführt werden. 
Verschiedene Fehler und Probleme können von den Beschäftigten in das System als Störungen eingetragen werden. Diese und auch mögliche Lösungen und Anmerkungen sind für andere Beschäftigte einsehbar. Liegt eine Störung vor, kann im ersten Schritt die Suchfunktion für Störungen genutzt werden, um bereits eingetragene Lösungsvorschläge zu erhalten und die Störung direkt innerhalb der Linie ohne eine zusätzliche Person zu lösen. Des Weiteren kann der Meister mithilfe des Assistenzsystems den Stand in der Linie überwachen und sogar hochgerechnete Vorhersagen der Kennzahlen in den einzelnen Stationen über die Schicht erzeugen.

\subsection{Projekthintergrund}

Die Digitalisierung der Produktion und der Produktionsarbeitsplätze hat unter dem Schlagwort Industrie 4.0 eine weitere Beschleunigung erfahren. Experten prognostizieren große Verbesserungspotentiale, v. a. im Bereich der Flexibilisierung und Individualisierung der Produkte und der zugehörigen Produktion. Beispielsweise wird von Einsparungen bei Bestands-, Fertigungs-, Logistik-, Komplexitäts-, Qualitäts- und Instandhaltungskosten ausgegangen [4]. Neben den neuen technischen Möglichkeiten zur Umgestaltung von Wertschöpfungsnetzwerken werden die Auswirkungen auf die Beschäftigten in den produzierenden Betrieben oft nur am Rande betrachtet. Jedoch werden die unter dem Begriff Industrie 4.0 beschriebenen Entwicklungen massive Auswirkungen auf die industrielle Arbeit, ihre Organisationsformen sowie die Kompetenzanforderungen der Belegschaften haben [1]. Für ein tieferes Verständnis der zu Grunde liegenden Wirkmechanismen hilft es, Produktion als sozio-technisches System darzustellen (siehe Abb. 5.2). Diese Darstellung erlaubt es, die Zusammenhänge zwischen den technischen, personellen, organisatorischen sowie strategischen Aspekten der Produktion systematisch darzustellen und zu analysieren.

Aufgrund der starken Wechselwirkungen genügt es nicht, die Elemente isoliert zu betrachten und die jeweiligen Teilbereiche lokal zu verbessern. Für ein ganzheitliches Verständnis des sozio-technischen Produktionssystems spielen vielmehr die Schnittstellen zwischen den genannten Elementen eine entscheidende Rolle.

Das Forschungsprojekt IntAKom (Intelligente Aufwertung der manuellen und teilautomatisierten Arbeit durch den Einsatz digitaler Kommunikationstechnologie) strebt die Entwicklung guter digitaler Assistenzsysteme für die Beschäftigten an. Durch die Assistenz soll die Leistungsfähigkeit der Beschäftigten in Bezug auf die Arbeitsaufgabe erhalten oder gar gesteigert werden. Das Ziel des Projektes ist die Entwicklung, Erprobung und Dokumentation eines übertragbaren Modells zur systematischen und ganzheitlichen Aufwertung von manuellen und teilautomatisierten Arbeitssystemen in der Produktion durch digitale Kommunikationstechnologie. Durch den Einsatz digitaler Medien als Assistenzsysteme sollen Möglichkeiten aufgezeigt werden, Arbeitsinhalte zu erweitern, anzureichern und innovative Arbeitsstrukturierungskonzepte wie Job-Rotation zu fördern. Darüber hinaus sollen die Beschäftigten stärker an der Beseitigung von 


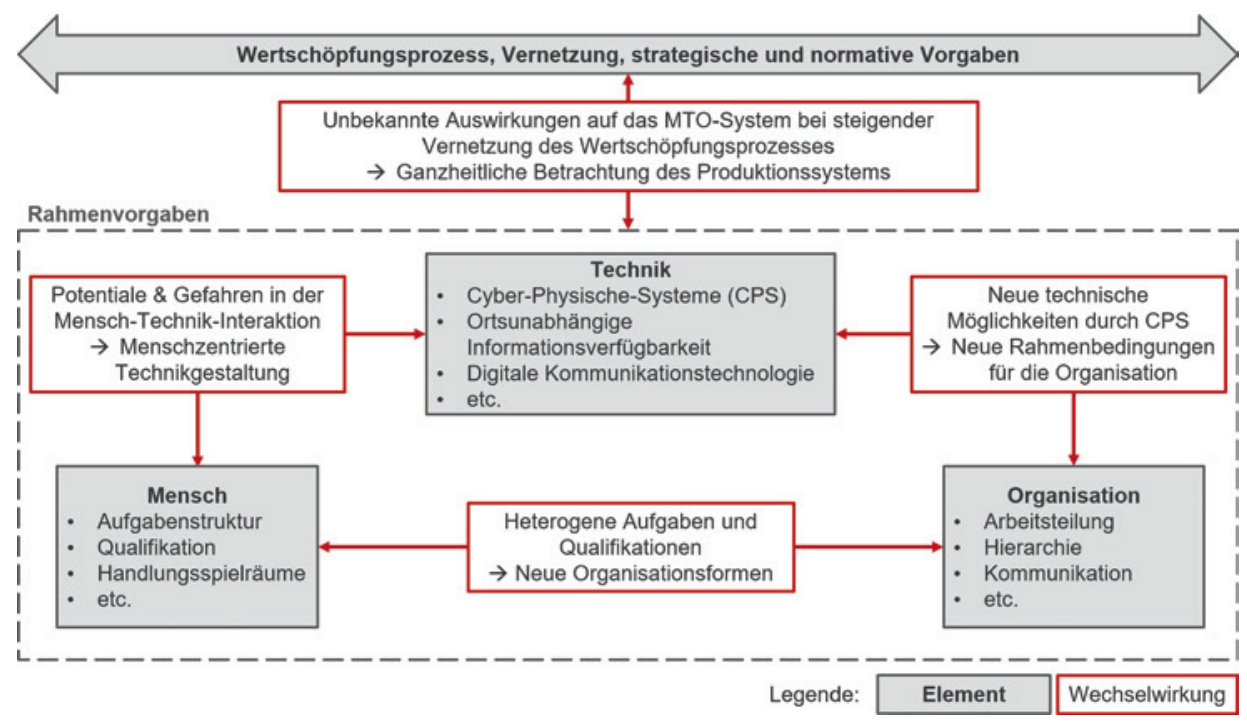

Abb. 5.2 Elemente und Wechselwirkungen in einem sozio-technischen System. (Eigene Darstellung in Anlehnung an [8] und [13])

Fehlern und Störungen beteiligt werden, und die dabei gewonnenen Erfahrungen strukturiert in die Verbesserung der Prozesse einfließen.

Die Interdisziplinarität der Thematik erfordert eine enge Zusammenarbeit unterschiedlicher wissenschaftlicher Fachgebiete. Das wissenschaftliche Konsortium, bestehend aus dem Fachgebiet für Arbeits- und Organisationspsychologie der Universität Kassel, der Gesellschaft für Personal- und Organisationsentwicklung und dem Institut für Produktionsmanagement, Technologie und Werkzeugmaschinen (PTW) der Technischen Universität Darmstadt, vereint das erforderliche Fachwissen in den Bereichen Mensch, Organisation, Technik und Wertschöpfungsprozess.

Neben der Kooperation zwischen Experten der wissenschaftlichen Disziplinen ist eine enge Zusammenarbeit von Wissenschaft und Industrie erforderlich, um technische und organisatorische Lösungen zur Aufwertung von Arbeit in der Produktion in die praktische Anwendung $\mathrm{zu}$ bringen. Die Expertise für den Einsatz moderner Kommunikationstechnologie und die softwareseitige Gestaltung der digitalen Medien steuert das auf Digitalisierungsprojekte spezialisierte Softwareunternehmen Bright Solutions bei. Die beteiligten Verbundunternehmen, deren Produktionsumgebungen im Rahmen des Projektes betrachtet werden, bringen das entscheidende Wissen zu den Produktionsprozessen und Beschäftigten mit. Bei der Zusammenstellung des Konsortiums wurden Unternehmen unterschiedlicher Branchen und Betriebsgrößen ausgewählt. Durch die Betrachtung der Fertigung eines KMU in der Elektronikbranche (mikrolab), der Produkte eines Werkzeugmaschinenherstellers (DATRON) sowie der Leuchtenendmontage eines Konzerns (Trilux) werden unterschiedliche 
Rahmenbedingungen und Zielstellungen berücksichtigt. Die Vielseitigkeit der Verbundunternehmen unterstützt die Interdisziplinarität des Vorhabens, da die jeweiligen Unternehmensvertreter eine hintergrundgeprägte und damit heterogene Sicht der Dinge auf die entwickelten Konzepte einbringen. Die Leitung des Projekts liegt beim PTW.

Das Gesamtprojekt umfasst im Wesentlichen vier Phasen. Zunächst wurden in jedem Unternehmen repräsentative Produktionsprozesse anhand einer interdisziplinären Analyse untersucht, um die Ist-Situation und Verbesserungspotenziale zu identifizieren. Anhand dieser Erkenntnisse wurden hinsichtlich vorab bestimmter Qualitätskriterien in jedem Verbundunternehmen spezifische Zielzustände definiert, um anschließend Konzepte zur komplementären Arbeitsgestaltung zu entwickeln. Die entwickelten Gestaltungsmaßnahmen wurden in Pilotprojekten in den Fertigungsumgebungen umgesetzt und evaluiert. Abb. 5.3 skizziert die Vorgehensweise des Forschungsprojektes:

Die These der Autoren ist, dass eine Aufwertung und Anreicherung der Arbeitsinhalte am Arbeitsplatz Shopfloor durch digitale Assistenzsysteme zur Erreichung der strategischen Ziele und somit zur Effizienzsteigerung des Wertstroms führt. Durch eine gute digitale Assistenz für Beschäftigte mit wertschöpfenden Tätigkeiten wird deren Leistungsfähigkeit und somit auch die des Wertschöpfungsprozesses gesteigert. Um diese These zu bestätigen, wurden unterschiedliche, repräsentative Arbeitsprozesse vor und nach der Aufwertung mittels einer quantitativen Nutzenbewertung analysiert. Für die definierten Arbeitsprozesse (z. B. Fertigung, Materialbereitstellung, Montage oder Problemlösung) wurden anschließend übertragbare Verbesserungsansätze entwickelt.

Die entwickelten Methoden sowie die gewonnenen Erkenntnisse aus dem Projekt wurden in einem Handbuch für ,gute digitale Assistenz“ zusammengefasst, um die Projektergebnisse für weitere Unternehmen nutzbar zu machen. Die übertragbaren und erprobten Methoden bilden hierbei neben den umgesetzten Lösungen die Basis des Handbuchs.

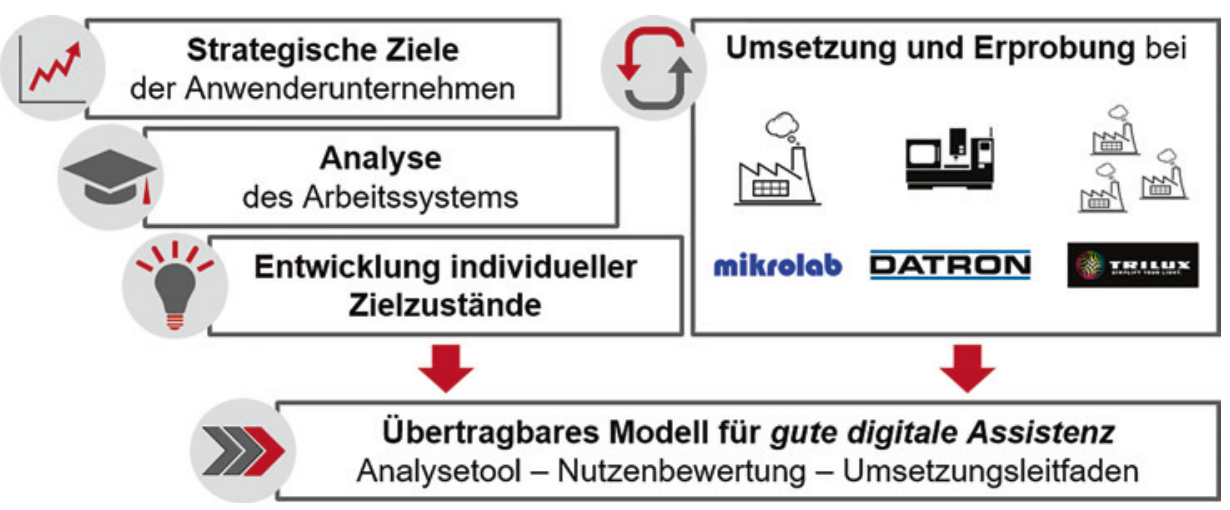

Abb. 5.3 Vorgehensweise beim Forschungsprojekt IntAKom. (Eigene Darstellung) 


\section{Vorgehensweise zur Zielerreichung}

Die Unternehmenspartner in dem Projekt IntAKom zielen mit der Entwicklung und Einführung eines digitalen Assistenzsystems auf die Steigerung der Produktivität der Montagebeschäftigten ab. Konkret sollen Fehler vermieden, die Qualität gesteigert und unproduktive Aufgaben reduziert werden. Der erste Schritt zur Einführung eines passgenauen digitalen Assistenzsystems ist die Analyse des Status-quo zur Identifikation der Bedarfe der betrachteten Unternehmen. Dem sozio-technischen Ansatz folgend wurden die Produktionsumgebungen der Verbundunternehmen aus Prozess-, Beschäftigten-, sowie organisationaler Sicht analysiert. Die angewandten Methoden wurden vorab zwischen den wissenschaftlichen Partnern aufeinander abgestimmt, um anschließend ein aussagekräftiges Gesamtbild zu erhalten.

Prozess (Informationsbedarfsanalyse) Für die zielorientierte Ausgestaltung eines digitalen Assistenzsystems ist die Berücksichtigung des zu unterstützenden Prozesses elementar. Um den Bedarf an Informationen für die produktiven Beschäftigten zu ermitteln, wurde von der TU Darmstadt die Informationsbedarfsanalyse entwickelt und bei den Unternehmenspartnern angewandt. Die Analyse von Informationsbedarfen legt den Grundbaustein für eine umfassende Informationsversorgung der Beschäftigten und somit auch zur Beherrschung komplexer Arbeitsaufgaben. Als Informationsbedarf wird die Art, Menge und Qualität der Informationen verstanden, die eine Person zur Aufgabenbewältigung zu einer bestimmten Zeit benötigt [9]. Bei der Analyse eines komplexen Produktionssystems ist eine möglichst umfangreiche Erfassung der vorhandenen Daten anzustreben. Die Reihenfolge der Anwendung sowie die direkten Zusammenhänge zwischen den Methoden im Rahmen der wertstromorientierten Informationsbedarfsanalyse sind in Abb. 5.4 dargestellt.

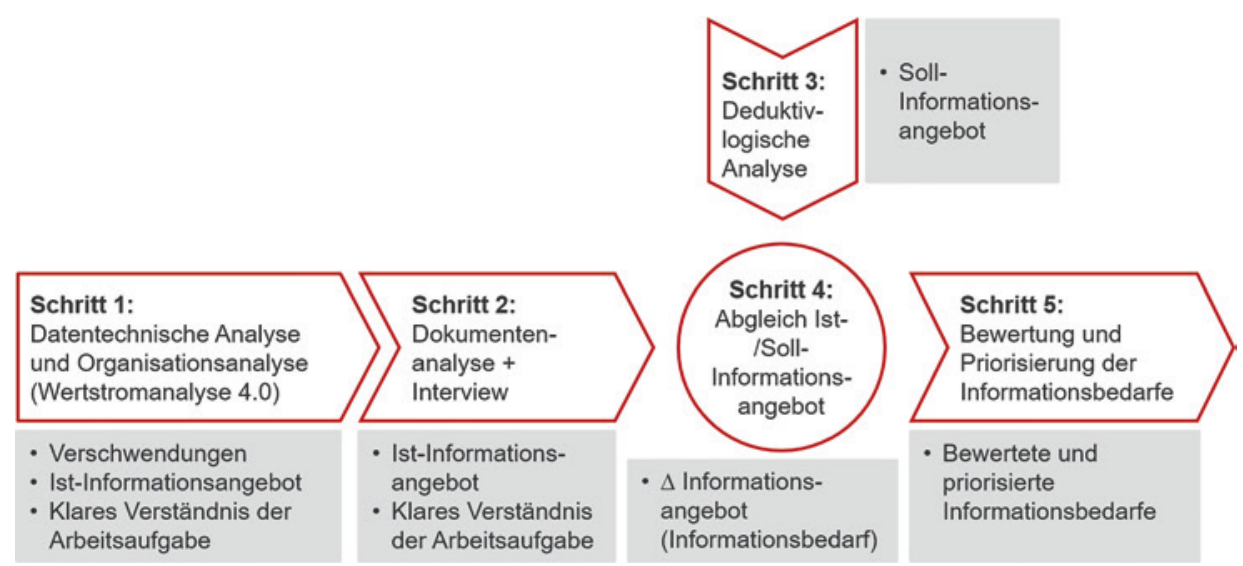

Abb. 5.4 Vorgehensweise bei der wertstromorientierten Informationsbedarfsanalyse. (Eigene Darstellung PTW) 
In fünf aufeinanderfolgenden Schritten werden Verschwendungen, die Arbeitsaufgabe, das Ist-Informationsangebot sowie das Soll-Informationsangebot erfasst, um daraus den Handlungsbedarf abzuleiten. Durch das strukturierte, chronologische Erfassen der Tätigkeiten der Beschäftigten im Rahmen der Arbeitsaufgabe lassen sich diesen auch die identifizierten Informationsbedarfe zuordnen.

Schritt 1, die betriebliche Datenerfassung (datentechnische Analyse und Organisationsanalyse), lässt sich anhand der am PTW entwickelten Methode „Wertstromanalyse 4.0 (WSA 4.0)“ durchführen [11]. Die Methode kombiniert die Analyse von Produkt- und Informationsflüssen mit der Analyse organisatorischer und strategischer Aspekte des Wertstroms einer Produktgruppe. Sie besteht aus sechs Schritten und erfasst neben den klassischen Arten der Verschwendung auch informationslogistische Verschwendungen. Somit wird die ganzheitliche Betrachtung von Wertstrom und Informationslogistik in der Produktion ermöglicht. Der Beobachter befindet sich zur Durchführung der Methode am Ort der Wertschöpfung und bewegt sich entlang der einzelnen Prozessschritte, wodurch er ein Verständnis des Gesamtprozesses sowie der Zusammenhänge zwischen einzelnen Tätigkeiten entwickelt. Im Rahmen der WSA 4.0 werden die Verschwendungen (in Form von nummerierten Kaizen-Blitzen) sowie das IstInformationsangebot dokumentiert. Zudem erhält der Beobachter ein klares Verständnis der Arbeitsaufgabe.

Schritt 2 umfasst die Dokumentenanalyse sowie gleichzeitig geführte Interviews mit den Aufgabenträgern, um die aktuell vorhandenen Informationen am Arbeitsplatz sowie den Zugriff der Beschäftigten auf diese Informationen zu erfassen. Bei der Dokumentenanalyse werden die aktuellen Informationsquellen, welche dem Aufgabenträger zur Verfügung stehen, analysiert [5]. Die Analyse der Dokumente wird idealerweise gemeinsam mit den Beschäftigten durchgeführt, indem diese zu den Dokumenten und Arbeitsabläufen interviewt werden. Da Schritt 2 im Anschluss an die Wertstromanalyse 4.0 durchgeführt wird und der Beobachter somit bereits ein umfassendes Prozessverständnis erworben hat, kann zu diesem Zeitpunkt bereits ein Zusammenhang zwischen Informationsangebot und -nachfrage herstellt werden. Diese Kenntnis fließt in Schritt 3 zur Definition des Soll-Zustandes ein.

Die deduktiv-logische Analyse in Schritt 3 überführt die Arbeitsaufgabe der Beschäftigten, ausgehend von deren Aufgaben und Zielen, sukzessive in Teilaufgaben. Von diesen Teilaufgaben können notwendige Informationen abgeleitet werden, die sich aus der jeweiligen Entscheidungssituation ergeben [10]. Nachdem im Rahmen der bisherigen Vorgehensweise überwiegend vorhandene Informationen betrachtet wurden, wird in diesem Schritt der Fokus auf die für die Arbeitsaufgabe benötigten Informationen gelegt. Der Beobachter hat die Aufgabe, notwendige Informationen für die Prozessschritte abzuleiten. Anhaltspunkte für zusätzlich benötigte Informationen können auch die im Rahmen der Wertstromanalyse 4.0 identifizierten Verschwendungen geben.

$\mathrm{Zu}$ diesem Zeitpunkt sollten die vollständigen Listen der aktuell verfügbaren sowie der idealerweise benötigten Informationen je Prozessschritt vorliegen. Aus der Differenz des Soll- und Ist-Informationsangebots (Schritt 4) ergibt sich ein konkreter 
Informationsbedarf. Dieser Bedarf kann eine zusätzlich benötigte Information, sowie die Korrektur von Inhalt (Art und Qualität), Quelle oder Bereitstellungszeitpunkt einer bereits vorhandenen Information sein.

Im letzten Schritt werden die Verschwendungen den identifizierten Informationsbedarfen zugeordnet. Dies erfolgt durch das Eintragen der Verschwendungen (nummerierte Kaizen-Blitze) in die letzte Spalte der Vorlage zur Dokumentation der Informationsbedarfe. Nicht jede im Rahmen der Wertstromanalyse identifizierte Verschwendung hat ihre Ursache in der Informationsversorgung. Andererseits führt die mangelnde Informationsversorgung fast immer zu Verschwendungen im Prozess. Durch die somit erfolgte Assoziation eines Informationsmangels mit einer negativen Auswirkung auf den Produktionsprozess kann eine qualitative Bewertung und Priorisierung der Informationsbedarfe vorgenommen werden.

Das Ergebnis der Informationsbedarfsanalyse ist anhand eines Beispiels in Abb. 5.6 dargestellt. Den Tätigkeiten in der entsprechenden Abfolge wird der Informationsbedarf in chronologischer Reihenfolge gegenübergestellt. Handlungsbedarfe werden mit einem Kaizen-Blitz sowie einer farblichen Hinterlegung gekennzeichnet, welche mögliche Verbesserungspotentiale darstellen. Beispielhaft werden in Abb. 5.6 zwei Kaizens aufgezeigt und entsprechend erläutert.

Beschäftigte (Fragebogen+Motion-Capturing mit Eye-Tracking) Für den Einsatz von digitalen Assistenzsystemen müssen in den Unternehmen Arbeitsmittel und Arbeitsbedingungen untersucht werden. Mithilfe des von der Universität Kassel - Fachgebiet Arbeits- und Organisationspsychologie (A\&O) - eingesetzten Systems „cEYEberman“ wird eine objektive Erfassung von Körperhaltungen und Blickdynamik zur Analyse von Arbeitssystemen, Verhaltensprävention und ergonomischer Gestaltung ermöglicht [3]. Die Positionierung des digitalen Assistenzsystems kann dadurch überprüft und ggf. angepasst werden. Das System vereint Inertialsensoren (Gyroskope, Accelerometer, Magnetometer) und Blickbewegungserfassung in einem am Körper getragenen Anzug (Abb. 5.7.) Es wird eine Bewertung generiert, die den Anteil ungünstiger Körperhaltungen dokumentiert, welche durch ein Zusammenwirken zwischen dem digitalen Assistenzsystem und den Arbeitsstationen entstehen können. Somit kann die ergonomische Bewertung der Arbeitsstation mit dem digitalen Assistenzsystem durchgeführt werden [15]. Es werden vor allem die aufgenommenen Daten der Blickbewegung genutzt, um störende Unterbrechungen des Arbeitsflusses durch das digitale Assistenzsystem zu erkennen. Durch diese Unterbrechungen können psychische Belastungen auftreten, da sie im Konflikt mit der Zielerreichung stehen [12] \& [14]. Um die Arbeitsbedingungen zu analysieren, sollten zwei unterschiedliche Erhebungsinstrumente verwendet werden. Zum einen der Fragebogen zur „Modularen Analyse von Belastungen in Organisationen“ (MABO) und zum anderen der Lernförderlichkeitsfragebogen (LFI). Der MABO erfasst die Einstellungen der Organisationsmitglieder zu den Themengebieten Organisationsklima, Führungsverhalten, Belastungen und der Mitarbeiterzufriedenheit. Angesichts aktueller Veränderungen in der Arbeitswelt werden die Themengebiete fortlaufend angepasst und weiterentwickelt [7]. 
Beim LFI handelt es sich um ein Beobachtungsinterview, mit dem systematisch Lernpotenziale von Arbeitstätigkeiten erfasst und ausgewertet werden [6]. Für den Grad der Lernförderlichkeit von Tätigkeiten, der zu einem maßgeblichen Wettbewerbsvorteil für Unternehmen wird [6], sind Merkmale wie z. B. Variabilität, Komplexität, Kommunikation/ Kooperation, Feedback, Information, Partizipation und Selbstständigkeit maßgeblich. Beide Instrumente wurden am Fachgebiet A\&O entwickelt und anhand von Gütekriterien überprüft. Die aufgeführten Instrumente wurden zum Zweck einer Prä-/ Post-Messung in den drei Verbundunternehmen eingesetzt und systematisch ausgewertet. Anschließend wurden die Ergebnisse in die jeweiligen Organisationen zurückgemeldet.

Organisatorisch (Interviews) Zur Erfassung der organisationalen Rahmenbedingungen wurden strukturierte Interviews eingesetzt. Fokus der Interviews war das Thema „Kultur und Führung“ mit den untergeordneten Themenfeldern Werte, Kommunikation, Beteiligung, Lernen/Qualifikation, Veränderung und Digitalisierung. Die Ergebnisse aller eingesetzten Analysemethoden wurden abschließend ausführlich mit Beschäftigten, Führungskräften und Arbeitnehmervertretern besprochen.

In den einzelnen Themenfeldern konnten im Hinblick auf die Organisation einige Anhaltspunkte zur Verbesserung bzw. zur notwendigen Veränderung identifiziert werden. Andererseits wurden Themenfelder identifiziert, die sich nicht verändern dürfen, wie z. B. große Handlungsspielräume und eigenverantwortliches Handeln, menschlich-familiäres Betriebsklima, Vertrauen und Offenheit.

Veränderungs- und Anpassungsbedarfe wurden im Unternehmen in der Kommunikation und den Kommunikationsmedien gesehen. Informelle Informationsund Kommunikationsformen haben an Bedeutung verloren; es wurden unterschiedliche Kommunikationsmedien verwendet, die aufgrund der teilweise vorliegenden Redundanz als störend empfunden wurden. Die Wünsche an das Assistenzsystem wurden klar formuliert: transparente Information, mehr Rückkopplungsschleifen zwischen den Abteilungen und mehr Vernetzung bei der Kommunikation.

Zusätzlich konnte herausgearbeitet werden, dass die Veränderungsprozesse vor allem im Bereich Prozesse und Strukturen weiter zu professionalisieren sind. Technische Innovationen und Veränderungen werden im Unternehmen akzeptiert, interne Veränderungen hinken dieser Akzeptanz hinterher. Bereits bekannte Herausforderungen, wie die fehlende Dokumentation von alten Prozessen, wurden weiter detailliert, um anschließend mögliche Lösungen zu finden.

Kriterien für eine lernförderliche Arbeitsgestaltung Ein zentraler Aspekt der Human- und Gestaltungskriterien guter Arbeit ist eine lernförderliche Arbeitsgestaltung. Lernförderlichkeit der Tätigkeit entscheidet darüber, ob die Kompetenz, die Motivation, die Gesundheit und die Persönlichkeit der Beschäftigten sich in der Arbeit negativ verändern, erhalten bleiben oder entwickeln können. Ob eine Tätigkeit mit digitalen Assistenzsystemen lernförderlich gestaltet ist, lässt sich nach den folgenden Kriterien beurteilen: 


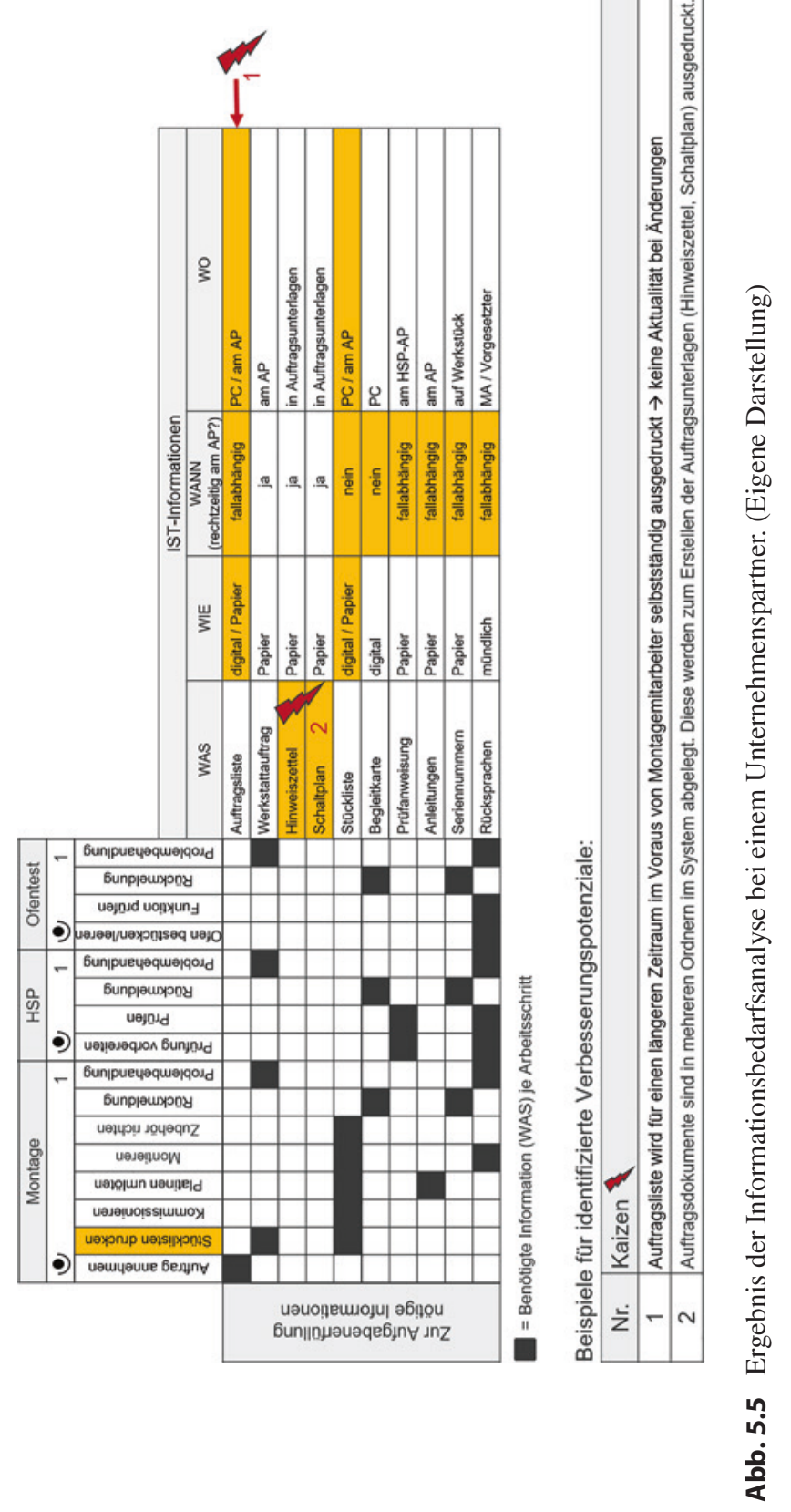


Abb. 5.6 Messsystem cEYEberman. (Eigene Darstellung (C) A\&O)

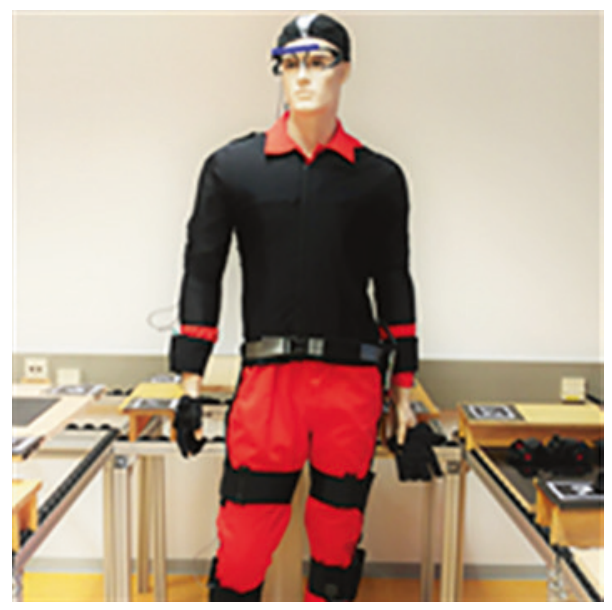

Tätigkeitsgestaltung - Ganzheitlichkeit: Ist die Tätigkeit vollständig? D. h. umfasst die Tätigkeit Planungs-, Vorbereitungs-, Ausführungs- und Kontrollanteile? Nicht immer sind diese vier Anteile in den einzelnen Tätigkeiten vorhanden. Eine Möglichkeit, diese Vorgabe zu erfüllen, besteht in einem Wechsel zwischen Tätigkeiten mit unterschiedlichen Anteilen der Vollständigkeit.

Handlungsspielräume (Vorgehen) - Autonomie: Wie hoch ist der Grad der Autonomie der Tätigkeit? Autonomie zielt in diesem Kontext auf die Handlungs- und Entscheidungsspielräume der Beschäftigten ab. Dabei steht der Begriff der Handlungsspielräume für die Möglichkeit, die Abarbeitung der Arbeitsaufträge zu variieren und kleinere Störungen selbst $\mathrm{zu}$ beheben. Entscheidungsspielräume beschreiben die Möglichkeit der Mitsprache bei der Verteilung von Arbeitsaufgaben sowie der Auswahl/ Beschaffung von Arbeitsmitteln.

Handlungsspielräume (zeitlich) - Arbeitsintensität: Lernen erfordert Zeit. Lernförderlichkeit ist dann gegeben, wenn eine geforderte Arbeitsmenge termin- und qualitätsgerecht erbracht werden kann und darüber hinaus die fachlichen und zeitlichen Ressourcen im erforderlichen Umfang zur Verfügung stehen.

Mitsprache - Mitwirkung: Diese beiden Kategorien adressieren sowohl die fachliche als auch die soziale Unterstützung bei der Ausführung schwieriger oder neuer Aufgaben durch die Führungskräfte und/oder Kollegen und die Frage, ob technisch-organisatorische Hilfsmittel zur Aufgabenerfüllung im erforderlichen Maße zur Verfügung stehen.

Transparenz - arbeitsbezogene Informationen: Beschäftigte brauchen zur Ausführung ihrer Tätigkeiten aktuelle Informationen über das Ziel und die Bedeutung ihrer Tätigkeit. Dieser Informationsbedarf bezieht sich sowohl auf die Bedeutung des 
Produktes für das Unternehmen allgemein als auch für den Kunden. Diese Informationen machen den Prozess als solches sicher und verbessern die Problemlösungs- und Innovationsmöglichkeiten der Beschäftigten.

Soziale Beziehungen - Interaktion stärkt die lernförderliche Arbeitsgestaltung. Sie kann Bestandteil der Tätigkeit sein (z. B. in der Zusammenarbeit mit Kollegen/ Kolleginnen, Führungskräften) oder durch organisatorische Maßnahmen (z. B. Qualitäts-/Gesundheitszirkel, systematischer Erfahrungsaustausch) realisiert werden. Genauso kann ein gezielter, gesteuerter Wechsel der Beschäftigten zwischen unterschiedlichen Arbeitsplätzen diesen Zweck erfüllen.

Lernen und Entwickeln - Qualifizierung: Neben dem impliziten Lernen im Prozess der Arbeit sind Angebote und Zugänge zu formalen Lernformen (Seminare, Kurse, Lehrgänge, Workshops, Zusatzausbildungen) wichtig. Hierdurch lassen sich fachliche und hierarchische Entwicklungswege gestalten und fachliche Sackgassen, die z. B. durch hochgradige Spezialisierung entstehen können, vermeiden.

Die nachfolgende Tab. 5.1 zeigt exemplarisch die positiven und negativen Wirkungen von Gestaltung digitaler Assistenzsysteme auf die Beschäftigten.

Entwicklung der Lösungen Die Ergebnisse der Analyse wurden in den Unternehmen mit allen Beteiligten strukturiert, geclustert und zu „Top-Themen“ zusammengefasst. So konnte die Gesamtaufgabe, Entwicklung und Einführung eines digitalen Assistenzsystems, in einzelnen Arbeitspaketen mit organisationalen oder technischen Schwerpunkten von den jeweiligen Experten bearbeitet werden.

Die identifizierten „Top-Themen“ sollen bei der Gestaltung, Einführung und Nutzung des zu entwickelnden digitalen Assistenzsystems adressiert werden. In den Unternehmen werden jeweils Arbeitsgruppen gebildet, um identifizierte Arbeitspakete zu bearbeiten. Die identifizierten „Top-Themen“ unternehmensübergreifend sind:

- Verantwortlichkeiten und Rollen,

- Beschäftigtenintegration bei Veränderungsprozessen,

- Informations- und Kommunikationsroutinen,

- Verfügbarkeit relevanter Informationen und Informationsbereitstellung

- und die Evaluation.

Das Ziel ist die Ausarbeitung konkreter Umsetzungskonzepte. Nach der Fertigstellung der Konzepte zu den spezifischen Themen in den einzelnen Unternehmen startet die Umsetzungsphase. Beispielhaft für ein Resultat der Konzeptphase ist in Abb. 5.8 das Ergebnis einer Informationsbedarfsanalyse sowie das daraus abgeleitete Mockup für das digitale Assistenzsystem dargestellt, welches als Diskussionsgrundlage zur Weiterentwicklung in Click-Dummys überführt werden kann. Diese von den 


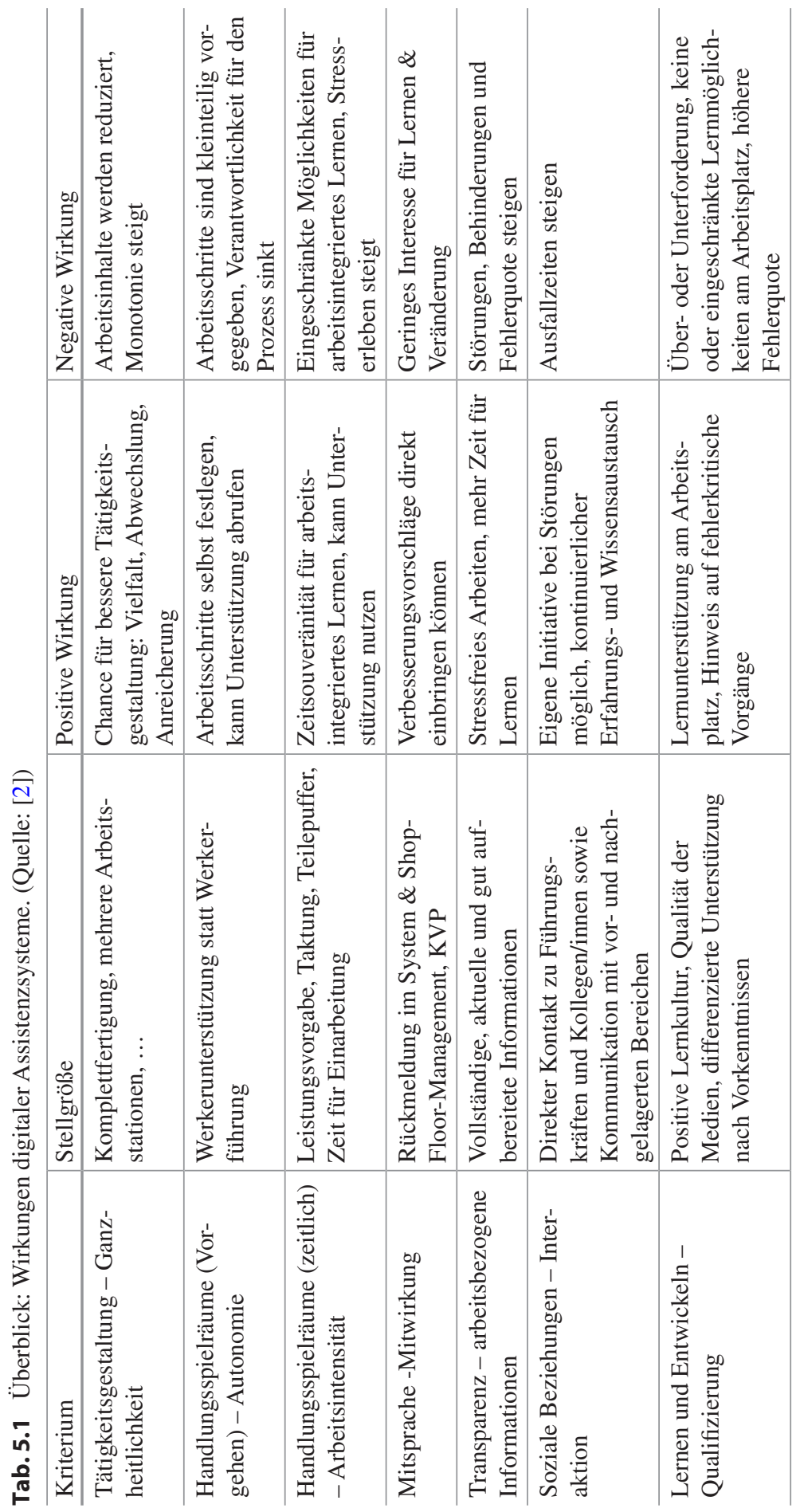


Maßnahmenliste aus der Informationsbedarfsanalyse

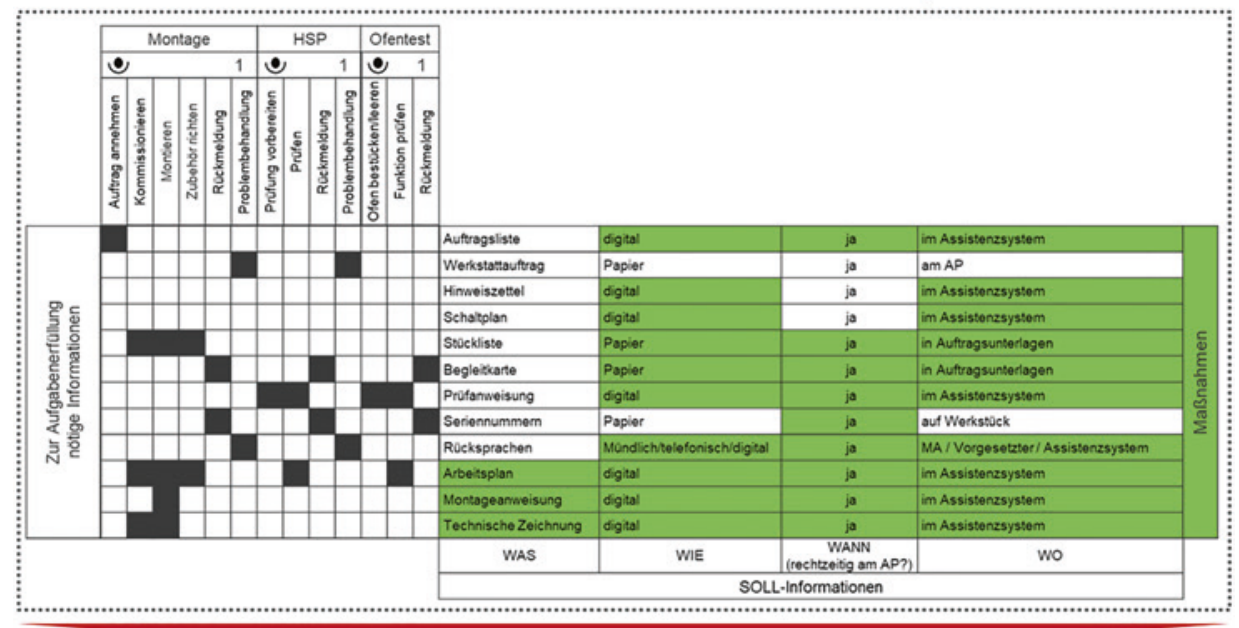

Mockup des Assistenzsystems

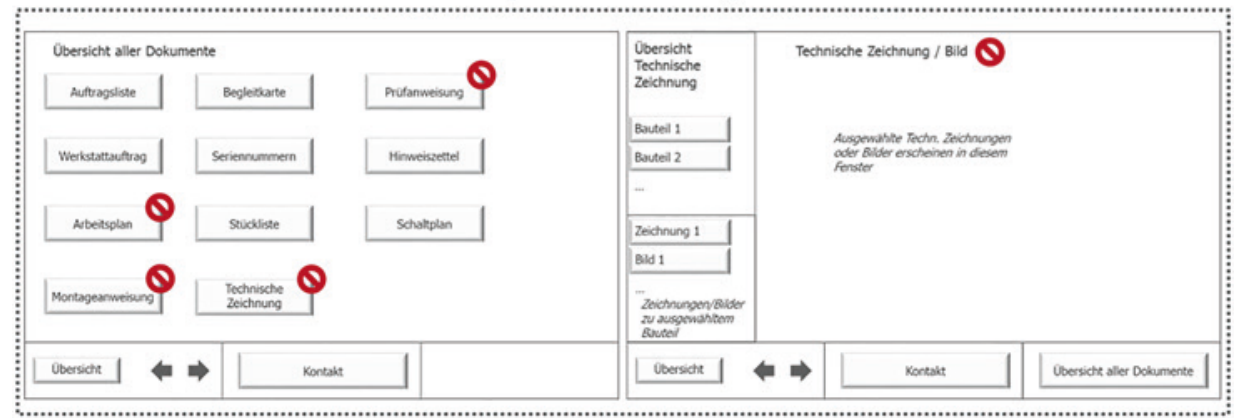

Abb. 5.7 Ableiten der Mockups aus den Ergebnissen der Informationsbedarfsanalyse. (Eigene Darstellung PTW)

Unternehmensvertretern bewerteten Prototypen des digitalen Assistenzsystems dienen als Grundlage zur Programmierung der Software.

Die Entwicklung des digitalen Assistenzsystems, das im Wesentlichen aus Software besteht, erfolgt entsprechend dem Branchenstandard iterativ in Sprints, indem der Zwischenstand in regelmäßigen Abständen von den Verbundunternehmen sowie dem beteiligten Software Unternehmen diskutiert wird. Als Grundlage für die effiziente Nutzung eines digitalen abteilungsübergreifenden Assistenzsystems sind klar definierte Prozesse und eindeutige Verantwortlichkeiten notwendig. Im Rahmen der Projektarbeit wurden diese Voraussetzungen gemeinsam mit den Unternehmen berücksichtigt. 


\subsection{Ergebnisse}

\subsubsection{Motion Capturing und MABO-Fragebogen}

Beim Motion Capturing des cEYEberman konnten, bezogen auf die Körperhaltung, keine Überschreitungen der Grenzwerte (Y-Achse 0-100\%) identifiziert werden. Die aufgenommenen Durchschnittswerte der Bewegungen des Beschäftigten bei einem der Verbundunternehmen sind in Abb. 5.8 dargestellt.

Innerhalb der Fragebögen wurde bei einem Verbundunternehmen deutlich, dass $80 \%$ der Beschäftigten in der Freizeit ein Smartphone und $88 \%$ andere digitale Medien nutzen. Bei der Post-Messung im März 2019 war die Smartphonenutzung auf $95 \%$ und die Nutzung der allgemeinen Medien auf $94 \%$ gestiegen. Die Affinität zur Digitalisierung ist besonders für die Einführung des digitalen Assistenzsystems von hoher Bedeutung und muss entsprechend berücksichtigt werden. Zudem konnten in Bezug auf die Einführung und Nutzung digitaler Assistenzsysteme auch kritische Punkte bei den Beschäftigten identifiziert werden.

So haben diese angegeben, dass sie eine zu starke Kontrolle befürchten und sich dadurch belastet fühlen. Auch Störungen und Unterbrechungen des Arbeitsablaufes wurde als Belastung benannt. Je mehr Störungen die Beschäftigten an ihrem Arbeitsplatz haben, desto negativer wird die Höhe der Bezahlung eingestuft und auch die Arbeitsbedingungen wurden kritischer bewertet. Die Ergebnisse zeigen, dass die Einführung eines digitalen Assistenzsystems gut durchdacht werden sollte, da kurz- und langfristige negative Effekte bei den Beschäftigten auftreten und auch die Zufriedenheit mit dem eingeführten Assistenzsystem beeinträchtigt werden kann. Aber auch positive Zusammenhänge wurden nach Einführung des digitalen Assistenzsystems in der Post-Messung

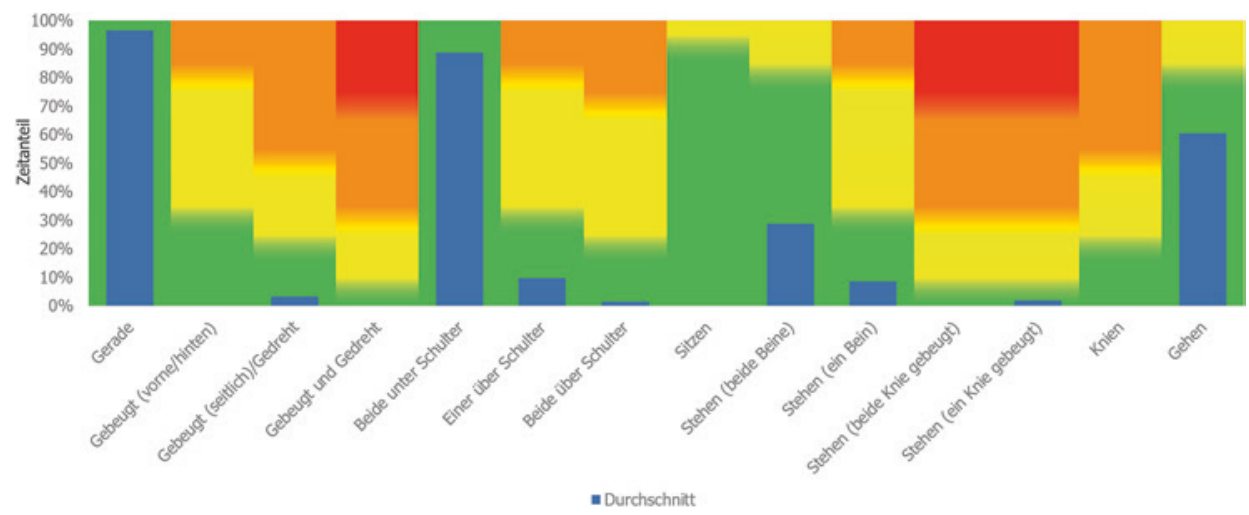

Abb. 5.8 Motion-Capturing der Arbeitspositionen. (Eigene Darstellung (C A\&O) 
deutlich. Die Beschäftigten betrachten Ihre Arbeit mit dem Assistenzsystem weiterhin als etwas Ganzes, Abgeschlossenes. Darüber hinaus wurden die Arbeitsinhalte als interessant und abwechslungsreich bewertet. Die ermittelten Informationen sind für grundlegende Aspekte der Funktionalität des Assistenzsystems relevant und können aufgrund der Befragungen bei den drei Verbundunternehmen berücksichtigt werden.

\subsubsection{Human- und Gestaltungskriterien für gute digitale Arbeit in der Montage}

Das beschriebene Verbundunternehmen (wie viele andere Unternehmen auch) stand vor der Herausforderung, die Digitalisierung in der Fertigung zu gestalten. Die Gründe für die Einführung eines digitalen Assistenzsystems waren:

- Hohe Komplexität in der Fertigung durch eine hohe Anzahl von Varianten

- Verkürzung der Lebenszyklen auf Baureihenebene

- Besserer Service durch Einbringen von Seriennummern in den Produkten

- Steigerung der Qualität und Senkung der Fertigungskosten

- Einführung neuer Geschäftsmodelle

- Aufwertung der Montagetätigkeit

Um die Auswirkungen des digitalen Assistenzsystems auf die Tätigkeitsgestaltung zu erfassen, wurden in dem Unternehmen die Einstellungen der Organisationsmitglieder zu den Themen Organisationsklima, Führungsverhalten, Belastungen und der Mitarbeiterzufriedenheit sowie den Lernpotenzialen der Beschäftigten mit zwei standardisierten Befragungsinstrumenten erfasst (vgl. Abschn. 5.2: Vorgehensweise zur Zielerreichung). Nach der Einführung des digitalen Assistenzsystems wurden durch Workshops mit Beschäftigten und Interviews mit betrieblichen Akteuren der Prozess und die Ergebnisse evaluiert. Gradmesser dieser Bewertung ist die menschengerechte Gestaltung von Arbeit ( 2 Arbeitsschutzgesetz, ArbSchG) mit den Merkmalen der lern- und gesundheitsförderlichen Arbeitsgestaltung [6]. Darüber hinaus wurden die digital ausgestatteten Arbeitsplätze mit dem Lernförderlichkeitsfragebogen (LFI) (vgl. ebenfalls Abschn. 5.2) analysiert.

Digitale Assistenzsysteme und Lernförderlichkeit Vor der Einführung des digitalen Assistenzsystems gab es z. T. manifeste Vorbehalte bei den Beschäftigten:

- Entgeltstruktur verschlechtert sich

- Qualifizierung wird vernachlässigt

- Soziales Miteinander wird eingeschränkt (,Teams fliegen auseinander“)

- Datenschutz wird vernachlässigt und die Leistungskontrolle nimmt zu (,gläserner MA“) 
Andererseits waren Hoffnungen mit der Einführung des digitalen Assistenzsystems verbunden:

- Selbstbestimmteres Arbeiten

- Weniger Störungen

- Beschäftigungssicherheit steigt

- Die Arbeit wird aufgewertet (z. B. durch den Umgang ,...mit modernen Arbeitsmitteln")

Die Evaluation hat gezeigt, dass sich die meisten Befürchtungen der Beschäftigten nicht bestätigt haben. Gründe hierfür liegen in der Arbeitsgestaltung mit den Aspekten der Lernförderlichkeit als zentralem Bestandteil der Human- und Gestaltungskriterien guter digitaler Arbeit, die nachfolgend exemplarisch reflektiert werden.

Der LFI wurde vor und nach der Einführung des digitalen Assistenzsystems eingesetzt. Hier wurde deutlich, dass die Selbstständigkeit vor Einführung des digitalen Assistenzsystems niedriger eingestuft wurde. Eine mögliche Erklärung könnte z. B. der Umgang mit Störungen sein. Die Beschäftigten können nach Einführung eines digitalen Assistenzsystems direkt im System nach Lösungsvorschlägen suchen, bevor der Einrichter oder Meister verständigt werden muss. Auch unbekannte Montageschritte können selbstständig nachgeschlagen und erlernt werden.

Tätigkeitsgestaltung: Lernförderliche Tätigkeiten sind in der Regel komplexer und erfordern daher erweiterte Kompetenzen, Informationen und Hilfsmittel. Digitale Assistenzsysteme können eine lernförderliche Tätigkeitsgestaltung unterstützen, wenn durch ihren Einsatz

- tätigkeitsspezifische Handlungsspielräume der Beschäftigten erweitert, zumindest aber erhalten werden,

- alle wesentlichen Arbeitsinformationen in geeigneter Weise am Arbeitsplatz zur Verfügung stehen,

- Informationen und Lerninhalte nutzerspezifisch aufbereitet werden,

- unterschiedliche Arbeitsniveaus (differentielle Arbeitsgestaltung) unterstützt werden,

- die Nutzung und der Aufbau von individuellem und organisationalem Erfahrungswissen gefördert wird sowie

- eine Verknüpfung zum betrieblichen KVP-Prozess hergestellt wird.

Das digitale Assistenzsystem hat an der durchzuführenden Tätigkeit nichts geändert (,Wir montieren nach wie vor“"); allerdings ist mit der Einführung eine Tätigkeit hinzugekommen: Scannen. Die Montage erfolgt nach wie vor in verschiedenen Arbeitsschritten, die mit der Einführung des digitalen Assistenzsystems stärker standardisiert worden sind. Aus Sicht der Beschäftigten haben sich aber keine veränderten körperlichen Anforderungen bzw. eine Über-/Unterforderung ergeben. 
Bezüglich der Handlungsspielräume dominiert bei den Beschäftigten die Wahrnehmung „Man fühlt sich selbstständiger, weil man selbst entscheiden kann und mehr Infos hat. Man muss nicht mehr die Einrichter fragen“. Die Leistungsvorgaben wurden im Zusammenhang mit der Einführung der digitalen Assistenz nicht angehoben, auch können die Arbeitsschritte nicht selbst festgelegt werden. Die Arbeit erscheint nun unabhängiger von anderen betrieblichen Akteuren (hier von den Einrichtern), denn das digitale Assistenzsystem erlaubt den Montierern den problemlosen Abruf von unterstützenden Funktionen. Gleichzeitig hat jedoch die Kommunikation mit den Einrichtern abgenommen.

Mitwirkung/Kommunikation: Mitwirkung und Kommunikation sind Aspekte, die unabhängig von digitalen Assistenzsystemen in der Unternehmenskultur verankert sein müssen. Im Verbundunternehmen war sowohl im Vorfeld der Einführung als auch in der Einführungsphase des Assistenzsystems der entscheidende Erfolgsfaktor die frühzeitige und sich regelmäßig wiederholende Kommunikation. Diese begann frühzeitig, war umfassend und kontinuierlich und wurde dialogorientiert geführt. Gab es im Vorfeld der Einführung manifeste Befürchtungen bei den Beschäftigten (,Wenn ich nicht mehr mitkomme, bleibe ich auf der Strecke“), konnten diese Befürchtungen dadurch abgemildert werden.

Im Verbundunternehmen wurde darauf geachtet, dass die Ideen und Vorschläge der Beschäftigten und der beteiligten operativen Führungskräfte mit in die Ausgestaltung des digitalen Assistenzsystems einfließen konnten. Regelmäßig gab es Rückmelderunden und die Möglichkeit für die Beschäftigten, eigene Ideen in den Prozess mit einzubringen.

Arbeitsbezogene Information: Das digitale Assistenzsystem bietet den Beschäftigten mehr Informationen direkt am Arbeitsplatz (,,mehr Informationen vor Ort - weniger Wege“). Durch das digitale Assistenzsystem sind Informationen direkt am Arbeitsplatz abrufbar: So können Montageanleitungen direkt über die EDV auf den Bildschirm abgerufen werden und müssen nicht mehr den Umweg über einen papiergebundenen Ausdruck nehmen, was für die Beschäftigten eine zeitliche Entlastung darstellt.

Lernmedien: Die Einführung des digitalen Assistenzsystems hatte erhebliche Auswirkungen auf die Qualifikation der Beschäftigten. Zwar sind nur im stark begrenzten Umfang Tätigkeitsinhalte weggefallen (die in erster Linie mit papiergebundenen Arbeiten im Zusammenhang standen), aber es sind neue hinzugekommen. Die Qualifizierungs- und Einarbeitungsphase fand in kleinen Gruppen in regelmäßigen Abständen statt. So früh wie möglich wurde mit dem System gearbeitet. Dabei wurde von den Beschäftigten besonders die Kompetenz und Geduld der Einarbeitenden hervorgehoben (,Gut und immer wieder erklärt“). Allerdings gibt es auch Hinweise darauf, dass ein Teil der Beschäftigten trotz der Schulungen Schwierigkeiten mit der digitalen Technik hatte.

Das digitale Assistenzsystem wurde im laufenden Montageprozess mit den damit verbundenen Herausforderungen eingeführt. Eine dieser Herausforderungen für die Beschäftigten war das Nachvollziehen der Änderungen am System. Regelmäßig und z. T. kurzzyklisch wurden Änderungen/Umstellungen am System vorgenommen. Dies 
im laufenden Montageprozess nachzuvollziehen, gelang den Beschäftigten nicht immer. Gleichwohl wird in der Summe das digitale Assistenzsystem überwiegend positiv beurteilt:

- „Arbeit abwechslungsreicher“

- „Mehr Flexibilität - mehr Sicherheit““

- „Ideen werden schneller umgesetzt“"

- „Mitwirkung bei der Optimierung““

Digitale Assistenzsysteme, Mitbestimmung und Partizipation Die Einführung, Nutzung und Optimierung von digitalen Assistenzsystemen muss durch einschlägige betriebliche Regelungen flankiert werden, in denen mindestens folgende Themen aufgegriffen werden müssen:

- Lern-, gesundheits- und persönlichkeitsförderliche Gestaltung der Arbeit

- Sicherheit im Wandel (z. B. Beschäftigung, Entgelt)

- Persönlichkeitsschutz (Datenschutz, Leistung und Kontrolle)

Darüber hinaus sollten Beschäftigte bei der Entwicklung, Erprobung, Nutzung und Weiterentwicklung des digitalen Assistenzsystems als Experten ihrer Arbeit einbezogen und ihre Vorschläge soweit wie möglich berücksichtigt werden.

Um die Wirkung von digitalen Assistenzsystemen auf die Arbeit zu bewerten bzw. digitale Assistenzsysteme zur Arbeitsgestaltung zu nutzen, stellt das Arbeitsschutzgesetz die Grundlage. Eine lernförderliche Arbeitsgestaltung ist eine explizite Maßgabe aus dem Arbeitsschutzgesetz inkl. Verordnungen/Betriebssicherheitsverordnung. Hierbei geht es auf der einen Seite um die Vermeidung von Fehlbeanspruchungen (Schutzfunktion) und auf der anderen Seite um eine lern-, gesundheits- und persönlichkeitsfördernde Arbeitsgestaltung (Präventionsfunktion).

Gefährdungsbeurteilung nach dem Arbeitsschutzgesetz Das Arbeitsschutzgesetz grenzt Gefährdungen deutlich von Gefahren ab: Gefährdung wird hier als ein Zustand oder eine Situation definiert, in der die Möglichkeit des Eintritts eines Gesundheitsschadens besteht. Nach dem Arbeitsschutzgesetz

- müssen alle mit der Arbeit verbundenen Gefährdungen ermittelt werden ( $\$ 5)$.

- Dabei ist der Stand der Technik, Arbeitsmedizin und Hygiene sowie gesicherte arbeitswissenschaftliche Erkenntnisse zu berücksichtigen ( $\$ 4)$.

- Gefahren sind an der Quelle zu bekämpfen und individuelle Schutzmaßnahmen sind nachrangig zu anderen Maßnahmen (§ 4).

- Maßnahmen sind mit dem Ziel zu planen, Technik, Arbeitsorganisation, sonstige Arbeitsbedingungen, soziale Beziehungen und Einfluss der Umwelt auf den Arbeitsplatz sachgerecht zu verknüpfen. 
Dabei beinhaltet diese Beurteilung einen vollständigen Handlungszyklus, der aus der Analyse, der Maßnahmenentwicklung und der Umsetzung, der Wirksamkeitskontrolle und der Optimierung des Vorgehens besteht. Alle Schritte müssen zudem dokumentiert werden.

Diese Gefährdungsbeurteilung muss in regelmäßigen Abständen wiederholt sowie bei technischen/organisatorischen Veränderungen neu durchgeführt werden.

In Verbindung mit dem Betriebsverfassungsgesetz hat die Arbeitnehmervertretung umfassende Mitbestimmungsrechte bei der Gefährdungsbeurteilung.

Leistungsüberwachung und Datenschutz Grundsätzlich hat der Arbeitgeber das Recht, seine Beschäftigten zu kontrollieren, denn er hat ein berechtigtes Interesse daran zu überprüfen, ob sich seine Beschäftigten an ihre arbeitsvertraglichen Pflichten halten. Dieses Kontrollrecht darf der Arbeitgeber allerdings nicht uneingeschränkt ausüben; er muss dabei folgende Grenzen beachten:

- Angemessenheit der Kontrollmaßnahme

- Persönlichkeitsrechte der Beschäftigten müssen beachtet werden (Grundgesetz)

- Grenzen durch den Datenschutz (DSGVO)

Nach $\S 80$ Abs. 1 Nr. 1 Betriebsverfassungsgesetz (BetrVG) hat in Unternehmen mit Betriebsrat dieser auf die Einhaltung der datenschutzrechtlichen Vorgaben zu achten. Er muss ebenfalls einbezogen werden, wenn Leistungsdaten erhoben werden. Dabei ist es unerheblich, ob diese Daten mittels technischer Hilfsmittel erhoben werden oder nicht; entscheidend ist die Speicherung und/oder Auswertung mittels EDV.

\subsubsection{Produktivität in der Produktion}

Um den Einfluss digitaler Assistenzsysteme auf die Produktivität eines Produktionssystems zu bestimmen, müssen zunächst konkrete und messbare Zielgrößen abgeleitet werden. Produktivität definiert sich in der Betriebswirtschaftslehre nach dem Verhältnis von Output- zu Inputmenge. Messbar ist die Produktivität als Quotient des Ertrages und des Faktoreinsatzes [17]. Abhängig von den betrachteten Input- und Outputfaktoren lassen sich unterschiedliche Kennzahlen errechnen, wie beispielsweise die Maschinenoder die Flächenproduktivität.

Da der Einfluss digitaler Assistenzsysteme bewertet werden soll, ist ausschließlich die Betrachtung der Arbeitsproduktivität (auch als Arbeitseinsatz bezeichnet) auf betriebswirtschaftlicher Ebene sinnvoll. Diese ist definiert als „die pro eingesetzter Einheit des Faktors Arbeit erzielte Produktionsmenge“" [16].

$$
\text { Arbeitsproduktivität }=\frac{\text { Ausbringungsmenge }}{\text { Arbeitseinsatzmenge }}
$$


Die Arbeitsproduktivität wird beeinflusst durch einen Verfügbarkeitsaspekt (ist das Arbeitssystem technisch einsatzbereit, sind Bediener bzw. Werker vorhanden, usw.), einen Leistungsaspekt (welche Normalleistung ist zu erwarten und wird diese Normalleistung gegenwärtig erreicht) sowie die Qualität der geleisteten Arbeit (sind die abgelieferten Teile/Produkte fehlerfrei oder fällt Ausschuss an bzw. ist Nacharbeit nötig). Insbesondere bei manueller Arbeit hängen sowohl die Leistung aber auch die Qualität vom Werker und damit die Produktivität als Ganzes ab.

Soll nun der Einfluss (oder auch Nutzen) digitaler Assistenzsysteme auf die Arbeitsproduktivität ermittelt werden, ist die Auswahl unmittelbar messbarer Kennzahlen notwendig. Diese Kennzahlen sollten möglichst nur durch das Assistenzsystem selbst und nicht durch Randeffekte beeinflusst werden. Zudem sollten ausgewählte Kennzahlen und die dafür benötigten Daten und Informationen kurzfristig und mit vertretbarem Aufwand aufgezeichnet werden können. So wird die praktikable Nutzung sowohl im Unternehmensalltag als auch zu Forschungszwecken gewährleistet. Weiterhin sollte darauf geachtet werden, dass keine Redundanzen bei aufzuzeichnenden Daten bzw. der Aussagekraft verschiedener Kennzahlen entstehen.

Diese Anforderungen werden erfüllt, indem die Arbeitsproduktivität auf Grundlage von konkreten und einfach messbaren Kennzahlen errechnet wird. In einem Umfeld mit Produkten, deren Arbeitsinhalte identisch oder ähnlich sind (meist hohe Stückzahlen und niedrige Typenvielfalt) lässt sich die Produktivität wie folgt messen:

$$
\text { Stundenleistung }\left[\frac{\text { Stück }}{h}\right]=\frac{\text { Stückzahl Gutteile }}{\text { Anwesenheitszeit MA }}
$$

Bei hoher Varianz der Arbeitsinhalte von Produkten, die über das gleiche Produktionssystem laufen, ist ein Vergleich von Produktivität auf der Basis produzierter Einheiten nicht aussagekräftig. Dies gilt v. a., da der Produktmix pro betrachteter Zeiteinheit variiert. Hier kann auf der Basis standardisierter Zeitvorgaben für jedes abgelieferte Stück (i. d. R. die Vorgabezeit aus der Arbeitsvorbereitung) auf den Anteil der produktiv verwendeten Anwesenheitszeit (,rückgemeldete Gutzeit“) ausgewichen werden:

$$
\text { Produktivzeitanteil[\%] }=\frac{\text { Produktivzeit MA }}{\text { Anwesenheitszeit MA }} 100 \%
$$

Um möglichst valide Ergebnisse zu erhalten, ist G1. 5.2 also vor allem für hohe Stückzahlen mit geringer Varianz und somit gleichbleibender Bearbeitungszeit geeignet, während hingegen G1 5.3 für niedrigere Stückzahlen mit hoher Varianz und häufig wechselnder Bearbeitungszeit verwendet werden kann.

Der Zähler in Gl. 5.2 errechnet sich aus der ermittelten Gesamtstückzahl abzüglich Ausschuss. Der Nenner erfasst die gesamte Anwesenheitszeit des Mitarbeiters. In Gl. 5.3 beschreibt die Produktivzeit die Zeit, in welcher die eigentliche Arbeitsaufgabe erledigt wird und der Mitarbeiter wertschöpfend tätig ist. Zeitaufwände für ungeplante Nacharbeiten, Stillstände/Störungen, Kommunikation oder das Informationshandling gelten als nicht wertschöpfend und werden gemeinsam mit der produktiven Zeit in der 
Anwesenheitszeit erfasst. Beide Formeln eigenen sich, um den Produktivitätszuwachs an einem Arbeitsplatz durch den Einsatz eines digitalen Assistenzsystems zu ermitteln.

Neben dem Potenzial für die Arbeitsproduktivität kann durch den Einsatz digitaler Assistenz eine Steigerung der Flexibilität der Beschäftigten und damit des Produktionsprozesses erreicht werden. Flexibilität ist ein zentrales Anforderungskriterium der industriellen Produktion. Für eine variantenreiche Produktion wird eine reaktionsschnelle und flexible Anpassung an externe und interne Gegebenheiten benötigt. Zudem bewirken gestiegene Kundenanforderungen und die Verstärkung des Wettbewerbs schwankende Mengenbedarfe. Diese Anforderungen erfordern eine arbeitsorganisatorische Umverteilung von Aufgaben und Verantwortlichkeiten zwischen den Beschäftigtengruppen. Ungelernte Arbeitskräfte können mittels digitaler Assistenz flexibler eingesetzt werden und Fachkräfte können zu einem größeren Zeitanteil Tätigkeiten mit höheren Anforderungen nachgehen. Dies wird mithilfe des Assistenzsystems durch eine bedarfsorientierte Informationsbereitstellung abhängig vom Ausbildungsgrad und dem Kenntnisstand der Beschäftigten ermöglicht. Letztendlich werden so Verschwendungen, Fehler und auch die benötigte Montagezeit im Arbeitsprozess reduziert und die Qualität erhöht.

Einflussmöglichkeiten digitaler Assistenz Da digitale Assistenz im Zusammenspiel mit dem Beschäftigten auf viele unterschiedliche Arten wirkt, ist der Einfluss auf die Produktivität schwer zu erfassen. Dennoch können verschiedene Einflussmöglichkeiten des Assistenzsystems aufgezeigt werden. Durch die Verfügbarkeit von leicht zugänglichen, verständlichen und korrekten Informationen am Arbeitsplatz lässt sich beispielsweise die Häufigkeit von Fehlern reduzieren. Zudem bieten Assistenzsysteme die Möglichkeit, Fehler durch den Einsatz von Sensoren besser zu erkennen. Mithilfe eines durch das System verbesserten Wissensmanagements ist der Beschäftigte so in der Lage, mehr Fehler zu beheben. In Summe lässt sich damit die Prozessqualität erhöhen. Weiterhin kann die Informationsversorgung zu einem reduzierten Aufwand (und damit einer Zeitersparnis) beim Informationshandling oder der Kommunikation führen, was den Zeitaufwand beim Ausführen der Tätigkeit, Kommissionieren, Transport, Rüsten oder Einarbeiten verringert. Werden diese Ziele erreicht, ist von einer geringeren Bearbeitungsdauer je Auftrag zu rechnen. Entscheidend ist jedoch eine auf den Anwendungsfall passende Ausgestaltung des Assistenzsystems. Weiterhin ist durch die Bereitstellung verständlicher Informationen eine Reduzierung der für die Arbeitsaufgabe benötigten Kompetenzen erreichbar. Somit ist eine größere Anzahl der Beschäftigten in der Lage, bestimmte Tätigkeiten auszuführen, was die Flexibilität des Arbeitssystems erhöht. Die beschriebenen Effekte beeinflussen die Produktivität des Arbeitssystems und sind somit relevant, um den Einfluss digitaler Assistenz zu erfassen.

Um die Auswirkungen digitaler Assistenz auf die Produktivität eines Produktionssystems messen zu können, ist neben der Definition der Zielgröße Arbeitsproduktivität zudem das digitale Assistenzsystem genauer zu definieren. Dies ist notwendig, um das betrachtete System beschreiben zu können. Zudem ist eine Abgrenzung zwischen unter- 
schiedlichen Systemen elementar, da sonst unterschiedliche Parameter miteinander verglichen werden. Mithilfe digitaler Assistenzsysteme ist eine sensorische und kognitive Unterstützung realisierbar. Innerhalb dieser beiden Kategorien lassen sich konkrete Funktionen abgrenzen, um Assistenzsysteme unterscheidbar zu machen. Die im Rahmen des Forschungsprojektes IntAKom definierten Funktionen sind in Abb. 5.9 dargestellt.

Auf Basis der beschriebenen Formeln für die Arbeitsproduktivität und der Funktionen digitaler Assistenz ist eine Quantifizierung der Produktivitätssteigerung grundsätzlich möglich. Durch das Überprüfen der Wirkung mit Prä-/Post-Messungen lassen sich korrelative Zusammenhänge zwischen einzelnen Funktionen und Zielgrößen messen, um somit neben der übergeordneten Forschungsfrage - Welchen quantifizierbaren Nutzen hat ein digitales Assistenzsystem auf die Leistungsfähigkeit von Montage-Beschäftigten? - auch untergeordnete Fragestellungen, wie beispielsweise nach der Höhe des Einflusses bzw. die gegenseitige Beeinflussung einzelner Funktionen, beantworten zu können.

Allerdings wird die Produktivität von weiteren Rahmenbedingungen beeinflusst. So ist zu erwarten, dass die Prozesseigenschaften (vor Allem das Produkt), das eingesetzte digitale Assistenzsystem sowie der Beschäftigte jeweils einen Einfluss auf die gemessene Produktivität haben. Bei der Durchführung von Prä-/Post-Messungen sind, abhängig von den beobachteten Beschäftigten und der Arbeitsaufgabe, sehr unterschiedliche Ergebnisse zu erwarten. Vereinfacht ist es möglich, Beschäftigte in zwei Gruppen zu unterteilen: Auf der einen Seite Personen, die bereits in der Lage sind, das betrachtete Produkt $\mathrm{zu}$ montieren (prozesserfahren), und auf der anderen Seite Personen, die die Montage zum ersten Mal durchführen sollen (prozessunerfahren). Die Arbeitsaufgabe (Montage des Produktes) ist - ebenso vereinfacht - in wenig, mittel und hochkomplexe Aufgaben unterteilt.

Die unterschiedlichen relevanten Einflüsse auf die beobachtete Produktivitätssteigerung durch den Einsatz digitaler Assistenz führen zu hohen Anforderungen an die Versuchsdurchführung. So sind verschiedene Versuchsgruppen, Arbeitsaufgaben (Produkte) und ein gezieltes Ein- und Ausschalten einzelner Funktionen des digitalen Assistenzsystems erforderlich. Zudem ist für aussagekräftige und statistisch relevante Ergebnisse eine hohe Anzahl an Wiederholungen erforderlich, was zu einem erheb-

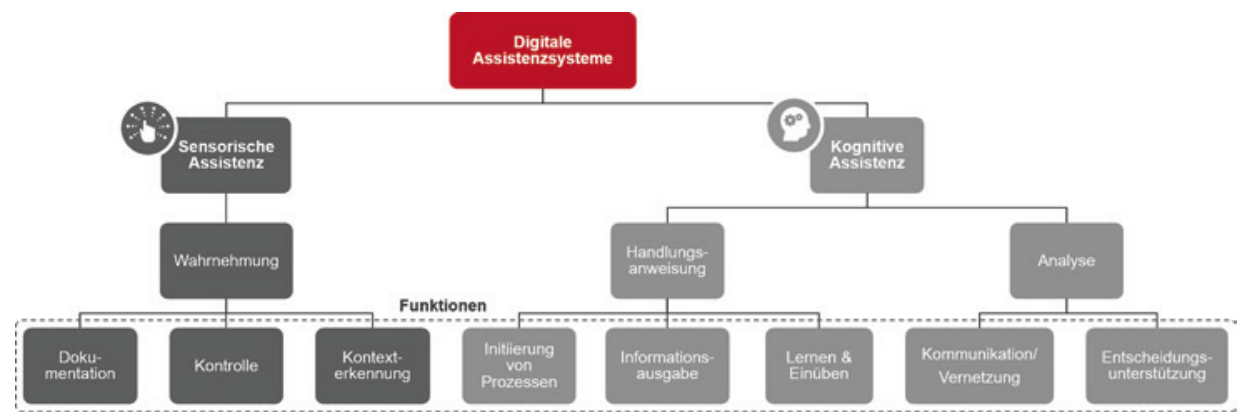

Abb. 5.9 Funktionen digitaler Assistenzsysteme. (Eigene Darstellung PTW) 
lichen Aufwand führt. Diese Umstände erschweren die quantitative Bewertung des Einflusses digitaler Assistenz im laufenden Betrieb. Zum Zeitpunkt des Schreibens dieses Beitrages ist es im Rahmen des Forschungsprojektes noch nicht gelungen, valide quantitative Ergebnisse in der Praxis zu ermitteln. Daher soll im Folgenden auf die qualitativ erfassten Auswirkungen digitaler Assistenz eingegangen werden. So konnten innerhalb des Projekts Auswirkungen sowohl auf den Produktionsprozess als auch auf die Informationsversorgung ermittelt werden, die als Bewertungsgrundlage dienen.

Qualitative Bewertung der Auswirkungen auf den Produktionsprozess Im Rahmen eines Workshops bei einem Projektpartner mit Beschäftigten, welche regelmäßig mit dem neu eingeführten digitalen Assistenzsystem arbeiten, wurden einige Vor- und Nachteile des Systems gesammelt und dokumentiert. Produktivitätsrelevante Vorteile sind im Folgenden aufgeführt:

- Bessere Verfügbarkeit technischer Informationen am Arbeitsplatz

- Optimierung des Gesamtprozesses durch eine automatische Programmierung der Steuerung am Arbeitsplatz

- Rückgang der Reklamationsquote

- Reduzierter Aufwand beim Handling von Daten

- Vereinfachte Nachbestellung von Material

- Vereinfachte Bearbeitung von Reklamationen

- Zeitersparnis beim Rüsten

An den Ergebnissen des Workshops wird deutlich, dass das eingeführte System den erwünschten Nutzen aus Sicht der Beschäftigten in vielen Punkten erfüllt. Bedingt durch die Einführung eines neuen Systems gibt es jedoch auch negative Effekte, die von den Beschäftigten angesprochen wurden. Diese Nachteile zeigen die empfundenen negativen Auswirkungen auf die Produktivität bei der Arbeit mit dem neuen System auf:

- Zeitlicher Aufwand bei der Bedienung des digitalen Assistenzsystems

- Flexibilitätseinbuße in der gesamten Montage während der Einführungsphase

- Überforderung einiger Beschäftigter aufgrund der umfangreichen Funktionen des Systems

Hier wird deutlich, dass ein wesentlicher Bestandteil eines neuen Systems die Einführungsphase ist. Für Unternehmen und deren Beschäftigte ist es daher besonders wichtig, einen transparenten Einführungsprozess zu gewährleisten, die Beschäftigten bei Entscheidungen mit einzubeziehen und ihr Feedback entsprechend wertzuschätzen. So können die genannten negativen Auswirkungen minimiert werden. Weiterhin wurden Vor- und Nachteile ohne einen direkten Bezug zur Produktivität des Montagesystems erfasst: 
- Organisatorische Vorteile

- Papierlose Montage

- Vereinfachte (untertägige) Kapazitätsplanung für die Meister

- Verbesserte Transparenz durch Auswertung von Daten

- Ermöglichung neuer Geschäftsmodelle (Verknüpfung von Serialnummern mit der Software der einzelnen Produkte - Traceability)

- Stärkere Abhängigkeit von der IT

- (Noch) Fehlende Schnittstellen zu anderen IT-Systemen

Mithilfe dieses Inputs kann das Unternehmen weitere Schritte in Richtung einer optimalen Einführung und stetigen Verbesserung eines digitalen Assistenzsystems gehen. Allerdings sind diese Punkte nicht relevant für die Betrachtung der Produktivität, welche hier im Fokus steht.

Qualitative Bewertung der Informationsversorgung Zudem bietet sich im Rahmen des Forschungsprojektes die Möglichkeit, die Informationsversorgung vor und nach dem Einsatz des neu entwickelten digitalen Assistenzsystems zu vergleichen. Anhand dieser Gegenüberstellung lässt sich eine deutliche Verbesserung der Informationsversorgung der Montagebeschäftigten erkennen (siehe Abb. 5.10). In der Gegenüberstellung ist gut zu erkennen, dass viele Informationen für die Beschäftigten der Montage vor Einführung des Assistenzsystems nicht oder nur schwer zu erhalten waren. Durch die gebündelte und einfache Bereitstellung der relevanten Informationen am Arbeitsplatz werden die Arbeitsbedingungen entschieden verbessert. Dies führt durch eine bedarfsorientierte Ausgestaltung bei den Projektpartnern zu den bereits aufgezeigten Einflussmöglichkeiten des digitalen Assistenzsystems.

\subsubsection{Beitrag zu übergeordneten Zielen}

Das Programm „Zukunft der Arbeit“ bildet eine von drei Säulen des Dachprogramms „Innovation für die Produktion, Dienstleistung und Arbeit von morgen“. Das Dachprogramm verbindet im Rahmen der neuen Hightech-Strategie die Teilbereiche Produktion, Dienstleistung und Arbeit in zukünftigen digitalisierten Umgebungen.

Mit der Digitalisierung von Herstellungsverfahren ändern sich auch in industriellen Produktionsumgebungen die Arbeitsinhalte und das Arbeitsumfeld der Beschäftigten. Gegenwärtige Konzepte autonomer Produktionssysteme, die auf eine weitgehende Automatisierung abzielen, orientieren sich vorrangig an wirtschaftlichen oder technologischen Kriterien. Merkmale einer lern- und gesundheitsförderlichen Arbeitsgestaltung spielen darin oft eine untergeordnete Rolle. Deshalb sind neue Ansätze der Gestaltung menschlicher Arbeit im Sinne der Beschäftigten erforderlich.

Im Projekt wurde Produktion in den Verbundunternehmen als sozio-technisches System begriffen, um die technischen, personellen, organisatorischen und strategischen 

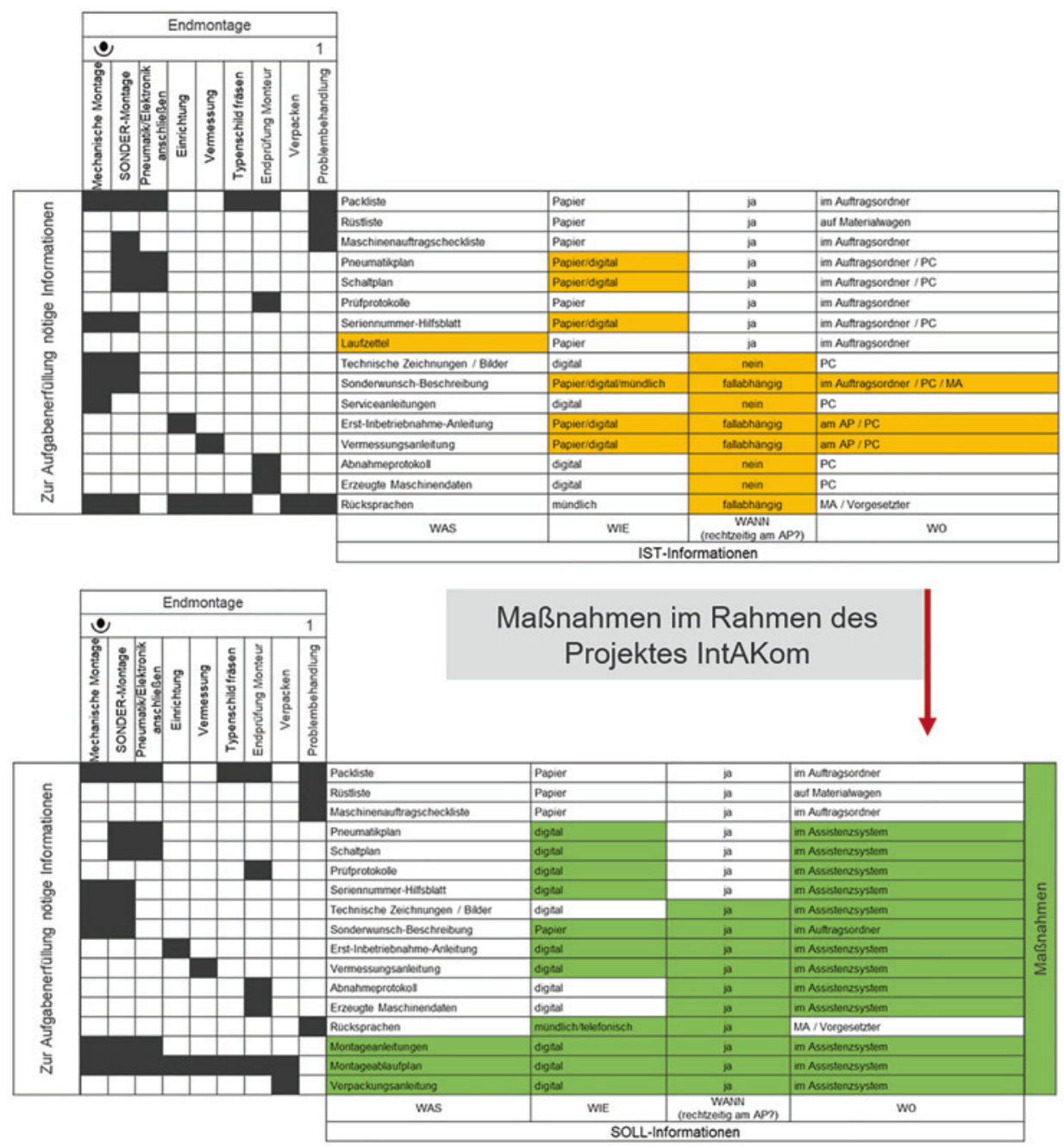

Abb. 5.10 Auswirkung digitaler Assistenz auf die Informationsversorgung. (Eigene Darstellung PTW)

Zusammenhänge zu analysieren und zu gestalten. Dieser Ansatz bezieht sich auf unterschiedliche Aspekte der drei thematischen Forschungs- und Entwicklungsbereiche, wobei der Schwerpunkt auf der „Ergonomischen und gesundheitsförderlichen Arbeitssystemgestaltung im digitalen Zeitalter" liegt.

Hier sind auch die Ziele des Projektverbundes und die Teilziele der Projektpartner verortet. Seitens der Ziele der beteiligten Institute geht es um die 
- Methode zur Definition von Einsatzmöglichkeiten, Überprüfung der technischen Umsetzbarkeit sowie einer systematischen Nutzenbewertung von Kommunikationstechnologien entlang des Wertstroms,

- Arbeits- und Tätigkeitsgestaltung, Kompetenzentwicklung und Lernen im Prozess der Arbeit auf Mitarbeiterebene, Auswirkungen von technologischen, organisatorischen und regulativen Veränderungen auf den Menschen und Unterstützung der Veränderungsprozesse,

- Analyse und Gestaltung von Arbeits- und Organisationskulturen bei der Aufwertung und lernförderlichen Ausgestaltung von Arbeit in der Montage mithilfe digitaler Informations- und Kommunikationsmittel.

Die komplementären Ziele der drei beteiligten Unternehmen im Verbund waren

- der systematische Ansatz zur Aufwertung teilautomatisierter oder manueller Arbeitsabläufe für das Arbeitssystem hinsichtlich ihrer Lernförderlichkeit und dessen Validierung als Pilotanwender für den Dienstleistungssektor im Bereich industrieller Produktion,

- die systematische Aufwertung teilautomatisierter oder manueller Arbeitsabläufe für das Arbeitssystem hinsichtlich ihrer Lernförderlichkeit und dessen Validierung als Pilotanwender in der variantenreichen Serienproduktion,

- die Entwicklung von systematischen Ansätzen zur Interaktion von Mensch und Maschine in den Lebenszyklusphasen Montage, Inbetriebnahme und Bedienung sowie bei der Fehleranalyse und - behebung mit dem Ziel der Unterstützung bei der Bedienung und des Lernprozesses.

Daneben ging es in einem kleineren Teil im Bereich „Digitalisierung der Arbeit als soziale Innovationschance“ um die Frage, wie eine Balance zwischen betrieblichen und auftragsbezogenen Zeit- und Leistungserwartungen in Einklang gebracht werden kann mit den Ansprüchen an Arbeit der Beschäftigten.

Die entwickelten Lösungen wurden in den drei Anwenderunternehmen implementiert. Abschließend fließen die im Rahmen des Projektes validierte Methodik sowie die implementierten Good-Pratice-Lösungen in ein Referenzmodell und eine übertragbare Vorgehensweise ein.

\subsection{Lessons learned}

Als Erfolgsfaktoren für die Einführung eines digitalen Assistenzsystems haben sich während der Projektlaufzeit eine Reihe von Aspekten herausgestellt, die nachfolgend näher beschrieben werden. 


\section{Vor Einführung eines digitalen Assistenzsystems}

\section{Klare Zielsetzung und Anforderungen an das digitale Assistenzsystem}

Vor Projektbeginn haben die Verbundunternehmen klare Ziele an das digitale Assistenzsystem definiert, die sich während der Laufzeit anpassen bzw. erweitern lassen. In den durchgeführten Workshops haben sich folgenden Zielstellungen an das digitale Assistenzsystem als Kernpunkte herausgestellt: Erhöhung der Produktivität, Unterstützung der Beschäftigten, lernförderliche Arbeit, Kundenwünsche realisieren, bessere Anpassung an neue Produkte, Qualifikation der Beschäftigten, Fehlerreduzierung, papierlose Produktion, weniger Reklamationen, mehr Daten, schnelleres Arbeiten, Qualitätsverbesserung und Überwachung, Minimierung von Störungen, selbstbestimmtes Arbeiten sowie Aufwertung der Arbeit und Umgang mit moderneren Arbeitsmitteln. Auch eine Kombination aus mehreren Zielen ist dabei denkbar. Im weiteren Verlauf des Projektes wurden die Ziele von den Unternehmensvertretern priorisiert und zur Fokussierung in eine Auswahl an „Top-Themen“ überführt. Anschließend wurde der Status-quo sowie der Zielzustand formuliert.

\section{Zusammensetzung der Arbeitsgruppe für die Umsetzung des digitalen Assistenz- systems \\ Von einer zuvor bestimmten Arbeitsgruppe sollte geklärt werden, inwieweit das} digitale Assistenzsystem intern oder extern programmiert wird. In dieser Arbeitsgruppe sollten nicht nur Führungskräfte, Abteilungsleiter oder andere Vertreter des mittleren Managements vertreten sein, sondern auch der Betriebsrat und eine Beschäftigtengruppe, die später mit den Assistenzsystemen arbeiten würde. Gerade der Betriebsrat sollte die bevorstehenden Veränderungen positiv nach Außen kommunizieren und den laufenden Prozess mitgestalten, um die Akzeptanz aller Beschäftigten zu erhöhen. Wichtig sind hierbei eine klare Aufgabenverteilung im Rahmen der Einführung und die Freistellung von Kapazitäten bei den betroffenen Beschäftigten. Während des Einführungsprozesses spielt die Führung der Arbeitsgruppe eine entscheidende Rolle.

\section{Information und Partizipation der Beschäftigten vor und während der Einführung eines digitalen Assistenzsystems}

Während aller Prozesse werden die Beschäftigten über die bevorstehenden Veränderungen informiert. So können frühzeitig Ängste genommen und die Beschäftigten auf die bevorstehenden digitalen Veränderungen vorbereitet werden. Der Mehrwert des digitalen Assistenzsystems muss den Beschäftigten aufgezeigt werden. In den Mitarbeiterbefragungen und Beschäftigteninterviews in den Verbundunternehmen wurde deutlich, dass sich die Beschäftigten mehr Partizipation und Mitsprache, Abwechslung, Verantwortung und Selbstständigkeit bei der Arbeit wünschen. Die Möglichkeit neue Dinge zu lernen, regelmäßig Informationen zu bekommen und gleichzeitig den Zusammenhalt und die Zusammenarbeit positiv zu fördern, wurde ebenfalls gewünscht. Schon bei der Funktionalität des Assistenzsystems sollten daher die Beschäftigten ein- 
bezogen werden. Sie kennen die alltäglichen Anforderungen (Spezialisten für die eigenen Arbeitsplätze) und können sehr hilfreiche Anregungen mit einfließen lassen. In dem Verbundunternehmen hat sich gezeigt, dass es erfolgsversprechend ist, das digitale Assistenzsystem während der einzelnen Entwicklungsphasen an einem Pilotarbeitsplatz zu testen und einen gezielten Schulungsplan zu entwickeln.

\section{Während der Einführung eines digitalen Assistenzsystems}

\section{Einarbeitung mittels Schulungsplan}

Erst nach der erfolgreichen Testphase werden die digitalen Assistenzsysteme an den einzelnen Arbeitsplätzen eingesetzt die Beschäftigten eingearbeitet und mittels Schulungsplan gezielt geschult. Dabei sollte die Einarbeitung der Beschäftigten direkt an den Arbeitsplätzen erfolgen. Für den laufenden Prozess können sogenannte Key-User ausgebildet werden, die bei aufkommenden Fragen oder Störungen Abhilfe leisten können. So wird nicht permanent die Führungskraft bei Rückfragen zum System benötigt. Die klare Definition von Rollen und Ansprechpartnern ist daher als weiterer Erfolgsfaktor anzubringen.

\section{Ergonomie}

Die grundsätzliche ergonomische Gestaltung der Arbeitsplätze darf bei der Einführung von digitalen Assistenzsystemen nicht außer Acht gelassen werden. In welchem Bereich des Arbeitsplatzes werden z. B. die Bildschirme angebracht. Auch die Zugänglichkeit für alle Beschäftigten muss gewährleistet werden.

\section{Nach Einführung eines digitalen Assistenzsystems}

\section{Veränderungen des digitalen Assistenzsystems nach einem bestimmten Zeitraum}

Veränderungen an dem implementierten Assistenzsystem sollten über einen gewissen Zeitraum gesammelt und $\mathrm{zu}$ vereinbarten Terminen eingepflegt werden, um die Beschäftigten nicht zu verwirren und Frustrationen zu vermeiden. Das digitale Assistenzsystem sollte für den laufenden Prozess bereits ein fortgeschrittenes Stadium erreicht haben.

\section{Klare Entgeltvereinbarungen}

Fehlen Vereinbarungen zwischen den betrieblichen Sozialpartnern über die Rahmenbedingen der digitalen Assistenzsysteme allgemein und/oder über die Einführung, wie z. B. Qualifizierung oder Entgelt, führt dies zwangsläufig zu Irritationen bei den Beschäftigten. 


\section{Literatur}

1. acatech (Hrsg) (2016) Kompetenzentwicklungsstudie Industrie 4.0 - Erste Ergebnisse und Schlussfolgerungen. https://www.acatech.de/publikation/kompetenzentwicklungsstudie-industrie-4-0-ersteergebnisse-und-schlussfolgerungen/. Zugegriffen: 10. Juli 2019

2. Anlauft W, Habenicht T (2018) Arbeit 4.0 - Ein Plädoyer für Weiterbildung und Arbeitsgestaltung. In: ffw $\mathrm{GmbH}$ (Hrsg) Zukunftsfähiges Kompetenzmanagement - prospektiv, lebensphasenorientiert und regional flankiert. Eigenverlag, Nürnberg, S 20-29

3. Arenius M, Klippert J, Ott A, \& Sträter O (2013) Ceyeberman: Ein Messsystem zur Ermittlung der Arbeitsbelastung [Ceyberman: A System for Measuring Physical and Cognitive Workload], EI - Der Eisenbahningenieur 64(7):36-39

4. Bauernhansl T (2014) Die Vierte industrielle Revolution. Der Weg in ein wertschaffendes Produktionsparadigma. In: Bauernhansl T, ten Hompel M, Vogel-Heuser B (Hrsg) Industrie 4.0 in Produktion, Automatisierung und Logistik. Springer Vieweg, Wiesbaden, S 31

5. Becker J, Winkelmann A (2014) Entwicklung einer Controlling- Konzeption. In: Becker J und Winkelmann A (Hrsg) Handelscontrolling. Springer, Berlin, Heidelberg, S 59-61

6. Bigalk D (2006) Lernförderlichkeit von Arbeitsplätzen - Spiegelbild der Organisation? Eine vergleichende Analyse (Englisch, 2018) von Unternehmen mit hoch und gering lernförderlichen Arbeitsplätzen. Zugl.: Kassel, Univ., Diss. Schriftenreihe Personal- und Organisationsentwicklung: Bd. 3. Kassel Univ. Press, Kassel

7. Englisch F (2018) MABO - Ein ganzheitliches Verfahren zur Gefährdungsanalyse von psychischer Belastung am Arbeitsplatz. In: Trimpop R, Kampe J, \& Bald M (Hrsg), 20. Workshop Psychologie der Arbeitssicherheit und Gesundheit: Voneinander lernen und miteinander die Zukunft gestalten!, S 725-728

8. Hirsch-Kreinsen H, Ittermann P, Niehaus J (2015) Digitalisierung industrieller Arbeit. Nomos Verlagsgesellschaft, Baden-Baden, S 15

9. Jung R (2006) Architekturen zur Datenintegration. Gestaltungsempfehlungen auf der Basis fachkonzeptueller Anforderungen. Deutscher Universitäts-Verlag (Wirtschaftsinformatik), Wiesbaden, $\mathrm{S} 51$

10. Jung R (2006) Architekturen zur Datenintegration. Gestaltungsempfehlungen auf der Basis fachkonzeptueller Anforderungen. Deutscher Universitäts-Verlag (Wirtschaftsinformatik), Wiesbaden, S 105

11. Meudt T, Metternich J, Abele E (2017) Value stream mapping 4.0: Holistic examination of value stream and information logistics in production. CIRP Annals - Manufacturing Technology $66: 413-416$

12. [13] Oesterreich R, Leitner K, Resch M (2000) Analyse psychischer Anforderungen und Belastungen in der Produktionsarbeit: Das Verfahren RHIA/VERA-Produktion. Hogrefe, Göttingen

13. Reinhart G, Bengler K, Dollinger Ch, Intra C, Lock Ch, Popova-Dlugosch S, Rimpau Ch, Schmidtler J, Teubner S, Vernim S (2017) Der Mensch in der Produktion von Morgen. Das Konzept Mensch - Technik - Organisation (MTO). In: Reinhart, G (Hrsg) Handbuch Industrie 4.0. Carl Hanser Verlag, München, S 55

14. Steffgen G (2004) Betriebliche Gesundheitsförderung: Problembezogene psychologische Interventionen. Psychologie für das Personalmanagement: Vol. 23. Hogrefe, Göttingen

15. Straeter O, Schmidt S, Stache S, Saki M, Wakula J, Bruder R, Glitsch U, Ditchen D (2018) Forschungsvorhaben „U-Linien-Montagesysteme“ U-Linien-Montagesysteme - Instrumente zur Gefährdungsbeurteilung und arbeitswissenschaftliche Gestaltungsempfehlungen zur Prävention.: Abschlussbericht BGHM 
16. Wirtschaftslexikon Arbeitsproduktivität. https://wirtschaftslexikon.gabler.de/definition/ arbeitsproduktivitaet-30245/version-253833. Zugegriffen: 30. Juli 2019

17. Wirtschaftslexikon Produktivität. https://wirtschaftslexikon.gabler.de/definition/produktivitaet46151/version-269437. Zugegriffen: 31. Juli 2019

Open Access Dieses Kapitel wird unter der Creative Commons Namensnennung 4.0 International Lizenz (http://creativecommons.org/licenses/by/4.0/deed.de) veröffentlicht, welche die Nutzung, Vervielfältigung, Bearbeitung, Verbreitung und Wiedergabe in jeglichem Medium und Format erlaubt, sofern Sie den/die ursprünglichen Autor(en) und die Quelle ordnungsgemäß nennen, einen Link zur Creative Commons Lizenz beifügen und angeben, ob Änderungen vorgenommen wurden.

Die in diesem Kapitel enthaltenen Bilder und sonstiges Drittmaterial unterliegen ebenfalls der genannten Creative Commons Lizenz, sofern sich aus der Abbildungslegende nichts anderes ergibt. Sofern das betreffende Material nicht unter der genannten Creative Commons Lizenz steht und die betreffende Handlung nicht nach gesetzlichen Vorschriften erlaubt ist, ist für die oben aufgeführten Weiterverwendungen des Materials die Einwilligung des jeweiligen Rechteinhabers einzuholen.

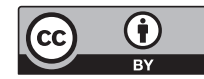

